\title{
Generalised Darboux-Koenigs Metrics and 3-Dimensional Superintegrable Systems
}

\author{
Allan P. FORDY ${ }^{\dagger}$ and Qing $H U A N G \ddagger$ \\ $\dagger$ School of Mathematics, University of Leeds, Leeds LS2 9JT, UK \\ E-mail: A.P.Fordy@leeds.ac.uk \\ $\ddagger$ School of Mathematics, Northwest University, Xi'an 710069, People’s Republic of China \\ E-mail: hqing@nwu.edu.cn
}

Received November 01, 2018, in final form April 16, 2019; Published online May 05, 2019

https://doi.org/10.3842/SIGMA.2019.037

\begin{abstract}
The Darboux-Koenigs metrics in 2D are an important class of conformally flat, non-constant curvature metrics with a single Killing vector and a pair of quadratic Killing tensors. In [arXiv:1804.06904] it was shown how to derive these by using the conformal symmetries of the 2D Euclidean metric. In this paper we consider the conformal symmetries of the 3D Euclidean metric and similarly derive a large family of conformally flat metrics possessing between 1 and 3 Killing vectors (and therefore not constant curvature), together with a number of quadratic Killing tensors. We refer to these as generalised Darboux-Koenigs metrics. We thus construct multi-parameter families of super-integrable systems in 3 degrees of freedom. Restricting the parameters increases the isometry algebra, which enables us to fully determine the Poisson algebra of first integrals. This larger algebra of isometries is then used to reduce from 3 to 2 degrees of freedom, obtaining Darboux-Koenigs kinetic energies with potential functions, which are specific cases of the known super-integrable potentials.
\end{abstract}

Key words: Darboux-Koenigs metrics; Hamiltonian system; super-integrability; Poisson algebra; conformal algebra

2010 Mathematics Subject Classification: 17B6; 37J15; 37J35; 70G45; 70G65; 70H06

\section{Introduction}

In recent years there has been a burst of activity in the identification and classification of superintegrable systems, both classical and quantum (see the review [14] and references therein). Most of the interest is in Hamiltonians which are in "natural form" (the sum of kinetic and potential energies), with the kinetic energy being quadratic in momenta. A non-degenerate kinetic energy is associated with (pseudo-)Riemannian metric, so the leading order term in any integral defines a Killing tensor for this metric, and itself commutes with the kinetic energy.

For a manifold with coordinates $\left(q_{1}, \ldots, q_{n}\right)$, metric coefficients $g_{i j}$, with inverse $g^{i j}$, the geodesic equations are Hamiltonian, with kinetic energy

$$
H=\frac{1}{2} \sum_{i, j=1}^{n} g^{i j} p_{i} p_{j}, \quad \text { where } \quad p_{i}=\sum_{k} g_{i k} \dot{q}_{k} .
$$

For a metric with isometries, the infinitesimal generators (Killing vectors) give rise to first integrals, which are linear in momenta (Noether constants).

The most common examples found in the literature have kinetic energies which are associated with flat or constant curvature metrics, so finding the leading order parts of higher order integrals (the Killing tensors) is straightforward, since, as is well known [8], they are all built as polynomial expressions in the first order integrals (Killing vectors). Indeed, in this case, (1.1) is actually the second order Casimir function of the symmetry algebra. However, for the non-constant 
curvature case, there is no algorithmic way known for building Killing tensors. In [6] a new method was proposed for the "next" class of metrics, namely the conformally flat case.

Conformally flat metrics possess an algebra of conformal symmetries, corresponding to the particular flat metric to which they are related. In 2 dimensions, as is well known, the conformal algebra is infinite. For $n \geq 3$ this algebra is finite and has maximal dimension $\frac{1}{2}(n+1)(n+2)$, which is achieved for conformally flat spaces (which includes flat and constant curvature spaces). Any two conformally equivalent metrics have the same conformal algebra, so we can describe this in terms of the corresponding flat metric. In flat spaces, the infinitesimal generators consist of $n$ translations, $\frac{1}{2} n(n-1)$ rotations, 1 scaling and $n$ inversions, totalling $\frac{1}{2}(n+1)(n+2)$. This algebra is isomorphic to $\mathfrak{s o}(n+1,1)$ (see [3, Vol. 1, p. 143]). Whilst the conformal algebra in two-dimensional spaces is infinite, there still exists the 6-dimensional subalgebra described above (with $n=2$ ).

The true symmetries (isometries) of a metric form a subalgebra of the conformal algebra. The maximum dimension of the algebra of isometries is $\frac{1}{2} n(n+1)$, which is realised when the space is flat or constant curvature. To discuss conformally flat metrics, we wish to avoid the flat and constant curvature cases, so should not have "too many isometries". In 2 dimensions we cannot have more than one Killing vector, since, by a theorem of Darboux and Koenigs, if such a metric possesses at least two Killing vectors, then it possesses three and the space has constant curvature (see [9, 10]). Killing vectors correspond to first integrals which are linear in momenta. Metrics found by Koenigs (see [9, 10, 11]) are characterised by having one linear and two quadratic first integrals. In modern day jargon, these are super-integrable systems, defined on spaces which are conformally flat, but not constant curvature. In [6] these systems were rederived by a new approach which builds higher order integrals as polynomial expressions in conformal symmetries, together with an important non-constant multiple of the Hamiltonian itself (see Section 3 below). The Darboux-Koenigs metrics have been further generalised in 2 dimensions, by considering Hamiltonians with one linear and one or more higher order first integrals (see [13] for the cubic case and [15] for integrals of any integer order).

In this paper, we consider the generalisation to 3 degrees of freedom. There exist $N$ dimensional generalisations of Darboux-Koenigs metrics, both in the classical and quantum domain $[1,2]$. In these papers the approach is more geometric and the connection to the 2dimensional Darboux-Koenigs metrics is different from the one presented below.

In Section 2 we describe the structure of the 10-dimensional conformal algebra in this case, and show that it has a particular decomposition introduced in [7]. We show that this algebra has 4 involutive automorphisms, which play an important role in our calculations. In Section 3 we describe the method introduced in [6] for the construction of (in this paper) quadratic first integrals out of conformal symmetries. We initially only assume the existence of one Killing vector $K$, but in 3 dimensions we can have up to 4 Killing vectors without forcing constant curvature (although the examples in this paper have at most 3 ). The calculations give rise to metrics which depend upon a number of parameters, and degeneration of these parameters leads to metrics with additional Killing vectors ("over-degeneration" leading to flat or constant curvature metrics). The significance of the additional Killing vectors is that we can use these to reduce from 3 to 2 degrees of freedom, using the "Kaluza-Klein reduction" approach described in [5]. This process leads to Hamiltonians in 2 degrees of freedom with one linear and two quadratic first integrals, which are Darboux-Koenigs kinetic energies with the addition of potential functions, which are compared with those of $[9,10]$. With enough Killing vectors, we can reduce in different ways, leading to the same Darboux-Koenigs kinetic energy, but different potentials. Within the 3-dimensional framework we give point transformations between these.

Another consequence of having more Killing vectors is that the Poisson algebra of first integrals is simplified, so that we can find the full set of relations. The involutive automorphisms play a crucial role here, since each algebra is invariant with respect to one of these. 
On the other hand, an example treated in Section 9.2 has only one Killing vector, so reduction to 2 dimensions results in a metric with no Killing vectors, but with higher order first integrals, thus leading to a maximally super-integrable system which falls out of the Darboux-Koenigs and later classifications.

The results on Poisson algebras are given in Sections 4 to 6 , whilst those on reductions can be found in Sections 7 to 10 .

\section{The 3D Euclidean metric and its conformal algebra}

Consider metrics which are conformally related to the standard Euclidean metric in 3 dimensions, with Cartesian coordinates $\left(q_{1}, q_{2}, q_{3}\right)$. The corresponding kinetic energy (1.1) takes the form

$$
H=\varphi\left(q_{1}, q_{2}, q_{3}\right)\left(p_{1}^{2}+p_{2}^{2}+p_{3}^{2}\right) .
$$

A conformal invariant $X$, linear in momenta, will satisfy $\{X, H\}=\lambda(X) H$, for some function $\lambda(X)$. The conformal invariants form a Poisson algebra, which we call the conformal algebra. For special cases of $\varphi\left(q_{1}, q_{2}, q_{3}\right)$ there will be a subalgebra for which $\{X, H\}=0$, thus forming true invariants of $H$. These correspond to infinitesimal isometries (Killing vectors) of the metric. Constant curvature metrics possess $\frac{1}{2} n(n+1)=6$ Killing vectors (when $n=3$ ).

As discussed in the introduction, the conformal algebra of this $3 \mathrm{D}$ metric has dimension $\frac{1}{2}(n+1)(n+2)=10$ (when $\left.n=3\right)$. A convenient basis is as follows

$$
\begin{aligned}
& e_{1}=p_{1}, \quad h_{1}=-2\left(q_{1} p_{1}+q_{2} p_{2}+q_{3} p_{3}\right), \\
& f_{1}=\left(q_{2}^{2}+q_{3}^{2}-q_{1}^{2}\right) p_{1}-2 q_{1} q_{2} p_{2}-2 q_{1} q_{3} p_{3}, \\
& e_{2}=p_{2}, \quad h_{2}=2\left(q_{1} p_{2}-q_{2} p_{1}\right), \\
& f_{2}=-4 q_{1} q_{2} p_{1}-2\left(q_{2}^{2}-q_{1}^{2}-q_{3}^{2}\right) p_{2}-4 q_{2} q_{3} p_{3}, \\
& e_{3}=p_{3}, \quad h_{3}=-2 q_{3} p_{1}+2 q_{1} p_{3}, \\
& f_{3}=-4 q_{1} q_{3} p_{1}-4 q_{2} q_{3} p_{2}-2\left(q_{3}^{2}-q_{1}^{2}-q_{2}^{2}\right) p_{3}, \\
& h_{4}=4 q_{3} p_{2}-4 q_{2} p_{3} .
\end{aligned}
$$

The Poisson relations of the ten elements in the conformal algebra (2.2) are given in Table 1. Note that this is an example of the conformal algebra given in [7, Table 3] corresponding to the case $a_{2}=a_{3}=2, a_{4}=0$. The subalgebra $\mathfrak{g}_{1}$, with basis $(2.2 \mathrm{a})$ is just a copy of $\mathfrak{s l}(2)$. We then make the vector space decomposition of the full algebra $\mathfrak{g}$ into invariant subspaces under the action of $\mathfrak{g}_{1}$ :

$$
\mathfrak{g}=\mathfrak{g}_{1}+\mathfrak{g}_{2}+\mathfrak{g}_{3}+\mathfrak{g}_{4}
$$

The basis elements for $\mathfrak{g}_{i}$ have the same subscript and are given in the rows of (2.2).

The algebra (2.2) possesses a number of involutive automorphisms:

$$
\begin{aligned}
& \iota_{12}:\left(q_{1}, q_{2}, q_{3}\right) \mapsto\left(q_{2}, q_{1}, q_{3}\right), \quad \iota_{13}:\left(q_{1}, q_{2}, q_{3}\right) \mapsto\left(q_{3}, q_{2}, q_{1}\right), \\
& \iota_{23}:\left(q_{1}, q_{2}, q_{3}\right) \mapsto\left(q_{1}, q_{3}, q_{2}\right), \\
& \iota_{e f}:\left(q_{1}, q_{2}, q_{3}\right) \mapsto\left(-\frac{q_{1}}{q_{1}^{2}+q_{2}^{2}+q_{3}^{2}},-\frac{q_{2}}{q_{1}^{2}+q_{2}^{2}+q_{3}^{2}},-\frac{q_{3}}{q_{1}^{2}+q_{2}^{2}+q_{3}^{2}}\right),
\end{aligned}
$$

whose actions are given in Table 2. 
Table 1. The 10-dimensional conformal algebra (2.2).

\begin{tabular}{|c||c|c|c||c|c|c||c|c|c||c|}
\hline & $e_{1}$ & $h_{1}$ & $f_{1}$ & $e_{2}$ & $h_{2}$ & $f_{2}$ & $e_{3}$ & $h_{3}$ & $f_{3}$ & $h_{4}$ \\
\hline \hline$e_{1}$ & 0 & $2 e_{1}$ & $-h_{1}$ & 0 & $-2 e_{2}$ & $-2 h_{2}$ & 0 & $-2 e_{3}$ & $-2 h_{3}$ & 0 \\
\hline$h_{1}$ & & 0 & $2 f_{1}$ & $-2 e_{2}$ & 0 & $2 f_{2}$ & $-2 e_{3}$ & 0 & $2 f_{3}$ & 0 \\
\hline$f_{1}$ & & & 0 & $-h_{2}$ & $-f_{2}$ & 0 & $-h_{3}$ & $-f_{3}$ & 0 & 0 \\
\hline \hline$e_{2}$ & & & & 0 & $2 e_{1}$ & $-2 h_{1}$ & 0 & 0 & $h_{4}$ & $4 e_{3}$ \\
\hline$h_{2}$ & & & & & 0 & $-4 f_{1}$ & 0 & $-h_{4}$ & 0 & $4 h_{3}$ \\
\hline$f_{2}$ & & & & & & 0 & $h_{4}$ & 0 & 0 & $4 f_{3}$ \\
\hline \hline$e_{3}$ & & & & & & & 0 & $2 e_{1}$ & $-2 h_{1}$ & $-4 e_{2}$ \\
\hline$h_{3}$ & & & & & & & & 0 & $-4 f_{1}$ & $-4 h_{2}$ \\
\hline$f_{3}$ & & & & & & & & & 0 & $-4 f_{2}$ \\
\hline \hline$h_{4}$ & & & & & & & & & & 0 \\
\hline
\end{tabular}

Table 2. The involutions of the conformal algebra (2.2).

\begin{tabular}{|c||c|c|c||c|c|c||c|c|c||c|}
\hline & $e_{1}$ & $h_{1}$ & $f_{1}$ & $e_{2}$ & $h_{2}$ & $f_{2}$ & $e_{3}$ & $h_{3}$ & $f_{3}$ & $h_{4}$ \\
\hline \hline$\iota_{12}$ & $e_{2}$ & $h_{1}$ & $\frac{1}{2} f_{2}$ & $e_{1}$ & $-h_{2}$ & $2 f_{1}$ & $e_{3}$ & $-\frac{1}{2} h_{4}$ & $f_{3}$ & $-2 h_{3}$ \\
\hline$\iota_{13}$ & $e_{3}$ & $h_{1}$ & $\frac{1}{2} f_{3}$ & $e_{2}$ & $\frac{1}{2} h_{4}$ & $f_{2}$ & $e_{1}$ & $-h_{3}$ & $2 f_{1}$ & $2 h_{2}$ \\
\hline$\iota_{23}$ & $e_{1}$ & $h_{1}$ & $f_{1}$ & $e_{3}$ & $h_{3}$ & $f_{3}$ & $e_{2}$ & $h_{2}$ & $f_{2}$ & $-h_{4}$ \\
\hline$\iota_{e f}$ & $-f_{1}$ & $-h_{1}$ & $-e_{1}$ & $-\frac{1}{2} f_{2}$ & $h_{2}$ & $-2 e_{2}$ & $-\frac{1}{2} f_{3}$ & $h_{3}$ & $-2 e_{3}$ & $h_{4}$ \\
\hline
\end{tabular}

\subsection{Different choices for the decomposition of $\mathfrak{g}$}

The first block of Table 2 shows different copies of $\mathfrak{s l}(2)$, which we might have chosen as our $\mathfrak{g}_{1}$. The remaining blocks are just the corresponding invariant subspaces.

In [7] we showed that the 6 -dimensional algebra $\mathfrak{g}_{1}+\mathfrak{g}_{2}$ (which here we call $\mathfrak{g}^{(0)}$ ) has two quadratic Casimirs

$$
\mathcal{C}_{2}^{(0)}=4 e_{1} f_{1}+h_{1}^{2}+2 e_{2} f_{2}-h_{2}^{2}, \quad \mathcal{C}_{4}^{(0)}=h_{1} h_{2}+e_{1} f_{2}-2 e_{2} f_{1},
$$

the second of which is derived from a quartic Casimir, which happens to be a perfect square in this case. In the $6 \times 6$ matrix representation (the adjoint representation), this is a multiple of the identity matrix, but in our Poisson representation, it vanishes identically, so the 6-dimensional Poisson algebra has a quadratic constraint.

Under the action of the involutions, this 6-dimensional algebra is transformed into the first 6 elements found to the right of $\iota_{i j}$ in Table 2 (here labelled $\mathfrak{g}^{(i j)}$ ), and the Casimirs take the form

$$
\begin{array}{ll}
\mathcal{C}_{2}^{(12)} & =\mathcal{C}_{2}^{(0)}, \quad \mathcal{C}_{4}^{(12)}=-\mathcal{C}_{4}^{(0)}, \\
\mathcal{C}_{2}^{(13)} & =2 e_{2} f_{2}+2 e_{3} f_{3}+h_{1}^{2}-\frac{1}{4} h_{4}^{2}, \quad \mathcal{C}_{4}^{(13)}=e_{3} f_{2}-e_{2} f_{3}+\frac{1}{2} h_{1} h_{4}, \\
\mathcal{C}_{2}^{(23)} & =4 e_{1} f_{1}+h_{1}^{2}+2 e_{3} f_{3}-h_{3}^{2}, \quad \mathcal{C}_{4}^{(23)}=h_{1} h_{3}+e_{1} f_{3}-2 e_{3} f_{1}, \\
\mathcal{C}_{2}^{(e f)} & =\mathcal{C}_{2}^{(0)}, \quad \mathcal{C}_{4}^{(e f)}=-\mathcal{C}_{4}^{(0)} .
\end{array}
$$

Each of the Casimirs $\mathcal{C}_{2}^{(0)}$ and $\mathcal{C}_{2}^{(i j)}$ represent spaces of constant curvature, whilst each of $\mathcal{C}_{4}^{(0)}$ and $\mathcal{C}_{4}^{(i j)}$ identically vanishes, so is a quadratic constraint on the whole 10-dimensional conformal algebra. 
There are 2 more 6-dimensional algebras:

$$
\mathfrak{g}^{(h e)}=\left\langle h_{2}, h_{3}, h_{4}, e_{1}, e_{2}, e_{3}\right\rangle, \quad \mathfrak{g}^{(h f)}=\left\langle h_{2}, h_{3}, h_{4}, f_{1}, f_{2}, f_{3}\right\rangle,
$$

with Casimirs

$$
\begin{aligned}
& \mathcal{C}_{2}^{(h e)}=e_{1}^{2}+e_{2}^{2}+e_{3}^{2}, \quad \mathcal{C}_{4}^{(h e)}=e_{1} h_{4}+2 e_{2} h_{3}-2 e_{3} h_{2}, \\
& \mathcal{C}_{2}^{(h f)}=f_{1}^{2}+\frac{1}{4}\left(f_{2}^{2}+f_{3}^{2}\right), \quad \mathcal{C}_{4}^{(h f)}=f_{3} h_{2}-f_{2} h_{3}-f_{1} h_{4},
\end{aligned}
$$

which are related through the involution $\iota_{e f}$. Again, $\mathcal{C}_{4}^{(h e)}$ and $\mathcal{C}_{4}^{(h f)}$ vanish identically.

\section{Geodesic flows in 3D with linear and quadratic integrals}

In this and later sections we seek Hamiltonian functions (2.1) which possess up to 4 first order invariants (Killing vectors), together with a number of quadratic integrals. In 3 dimensions, if we have 5 isometries, then we must have 6 , so the space has constant curvature. We already mentioned that in 2 dimensions, Darboux and Koenigs proved that we can only have 1 isometry (i.e., 2 implied 3). This "gap phenomenon" occurs in all dimensions and is the subject of [12].

Initially we only assume the existence of one Killing vector $K$, the calculations giving rise to metrics which depend upon a number of parameters. Degeneration of these parameters leads to metrics with additional Killing vectors, with "over-degeneration" leading to flat or constant curvature metrics. We use the method introduced in [6] to construct quadratic invariants out of conformal invariants.

A quadratic conformal invariant is any expression of the form

$$
F=\sum_{i, j=1}^{10} \beta_{i j} X_{i} X_{j}+\psi\left(q_{1}, q_{2}, q_{3}\right) H
$$

where $\beta_{i j}$ is a symmetric matrix of (constant) coefficients, $X_{i}$ are linear conformal invariants, and $\psi\left(q_{1}, q_{2}, q_{3}\right)$ is an arbitrary function, which satisfies

$$
\{F, H\}=\left(\sum_{i=1}^{3} \mu_{i}\left(q_{1}, q_{2}, q_{3}\right) p_{i}\right) H,
$$

where $\mu_{i}\left(q_{1}, q_{2}, q_{3}\right)$ are some functions.

We can ask whether there is a choice of $\beta_{i j}$ and $\psi\left(q_{1}, q_{2}, q_{3}\right)$ for which $\mu_{i}\left(q_{1}, q_{2}, q_{3}\right) \equiv 0$, in which case $F$ is a quadratic invariant. In fact, we have more structure. If both $K$ and $F$ are invariants, then so are $\{F, K\},\{\{F, K\}, K\}$, etc.

\subsection{Using the involutions}

It follows from the involutions of Table 2, that we can limit our choice of a single first order invariant to just 3 elements of our algebra: $e_{1}, h_{1}$ and $h_{4}$. Any other element is equivalent to one of these. Having fixed this invariant we choose a quadratic conformal invariant (3.1) with specific form

$$
F=X_{i} X_{j}+\psi\left(q_{1}, q_{2}, q_{3}\right) H
$$

where $X_{i}$ and $X_{j}$ are linear conformal invariants. At this stage we still have some equivalent choices from involutions which leave our choice of Killing vector invariant. For example, $e_{1}$ is fixed under the action of $\iota_{23}$, while $h_{1}$ is fixed (up to sign) by them all and $h_{4}$ is fixed (up to sign) by $\iota_{23}$ and $\iota_{e f}$. 


\subsection{Reductions to two dimensions}

In Sections 4, 5 and 6 we present a number of systems in 3 degrees of freedom, which have at least one first integral of degree one in momenta, as well as 3 independent quadratic integrals. These systems depend upon a number of parameters and, in full generality, the Poisson algebras are complicated, but by restricting parameters we can obtain systems with larger isometry algebras (first order integrals) and this enables us to calculate the full set of Poisson relations. The involutions play an important role in this calculation.

There is clearly an analogy with the Darboux-Koenigs systems in 2 degrees of freedom, but we show that there is a much deeper connection. The increased isometry algebra, obtained by restricting parameters, can be used to reduce the system from 3 to 2 degrees of freedom. After this process, we find that each of our systems reduces to one particular Darboux-Koenigs system, but with the addition of a potential. We compare the resulting potentials with those presented in $[9,10]$.

We use the particular method of reduction introduced in [5], referred to as the "KaluzaKlein reduction", since it is essentially the reverse procedure to the Kaluza-Klein extension. By adapting coordinates to a linear first integral, we can reduce from 3 to 2 degrees of freedom. In principle, the lower-dimensional system would possess vector potential terms, but in the examples of this paper (and of [5]) these can be removed by gauge transformation. Reduction to 2 degrees of freedom is not enough. We would like our system to be at least completely integrable and, preferably, maximally super-integrable. This requires the existence of integrals of the original system, which commute with the linear integral. We would like to choose our coordinate system so that the reduced kinetic energy is explicitly conformally related to the standard Cartesian kinetic energy. We would like to be able to construct any symmetries and a 6 -dimensional subalgebra of conformal symmetries of the corresponding 2-dimensional metric from invariant combinations of the conformal algebra (2.2). All of these things will be done for the systems discussed in this paper.

Each additional Killing vector allows us to reduce to 2 dimensions in a different way. From a given starting point, we always arrive at the same Darboux-Koenigs metric, but the specific reduction gives us a particular potential function. This allows us to relate different 2 -dimensional potentials by a "rotation" in 3 space.

\section{Systems with $K=e_{1}$}

In the case $K=e_{1}$, we consider the Hamiltonian (2.1) of the form

$$
H=\varphi\left(q_{2}, q_{3}\right)\left(p_{1}^{2}+p_{2}^{2}+p_{3}^{2}\right),
$$

and exploit the following chains of length 3 , found in the algebra:

$$
\left\{\cdot, e_{1}\right\}: f_{1} \mapsto h_{1} \mapsto-2 e_{1} \mapsto 0 \quad \text { and } \quad\left\{\cdot, e_{1}\right\}: f_{k} \mapsto 2 h_{k} \mapsto 4 e_{k} \mapsto 0, \quad k=2,3 .
$$

We use this to build 3 functions which satisfy $\left\{\cdot, e_{1}\right\}: F_{1} \mapsto F_{2} \mapsto F_{3} \mapsto 0$. If we can find $\varphi$ and $F_{1}$, such that $\left\{F_{1}, H\right\}=0$, then, by the Jacobi identity, we automatically have $\left\{F_{2}, H\right\}=$ $\left\{F_{3}, H\right\}=0$.

\section{1 $\quad F_{1}=e_{2} f_{2}+\psi\left(q_{1}, q_{2}, q_{3}\right) H$}

We consider a metric (4.1) with the chain of functions generated by $F_{1}=e_{2} f_{2}+\psi\left(q_{1}, q_{2}, q_{3}\right) H$, under the action of $e_{1}: F_{2}=\frac{1}{2}\left\{F_{1}, e_{1}\right\}, F_{3}=\frac{1}{2}\left\{F_{2}, e_{1}\right\}$, namely,

$$
F_{1}=e_{2} f_{2}+\psi H, \quad F_{2}=e_{2} h_{2}+\frac{1}{2} \psi_{q_{1}} H, \quad F_{3}=e_{2}^{2}+\frac{1}{4} \psi_{q_{1} q_{1}} H .
$$


The conditions $\left\{F_{1}, H\right\}=\left\{F_{3}, e_{1}\right\}=0$ lead to

$$
\varphi=\frac{q_{2}^{2} q_{3}^{2}}{\alpha q_{2}^{2}+\beta q_{3}^{2}+\gamma q_{2}^{2} q_{3}^{2}}, \quad \psi=-2 \beta \frac{q_{1}^{2}+q_{3}^{2}}{q_{2}^{2}}+2 \gamma q_{2}^{2} .
$$

Since $H, e_{1}, F_{1}, F_{2}, F_{3}$ are independent first integrals of $H$, this Hamiltonian is maximally super-integrable. We also have that $H, e_{1}, F_{3}$ are in involution.

Remark 4.1 (the Stäckel transform). The Hamiltonian (4.1), with $\varphi$ given by (4.3) can be regarded as the Stäckel transform of the Euclidean kinetic energy with potential $V=\varphi^{-1}$. Such potentials were classified in [4] and this example corresponds to a reduction of $V_{[3,1,1]}$, given in [4, Section 5], by equating $\left(q_{1}, q_{2}, q_{3}\right)=(x, y, z)$ and $(\alpha, \beta, \gamma)=\left(a_{3}, a_{2}, a_{5}\right)$.

Notice that the resulting metric is invariant under the involution $\iota_{23}$ (extended to include $\alpha \leftrightarrow \beta$ ), so we also have the integrals $G_{i}=\iota_{23}\left(F_{i}\right)$. These are not, of course, functionally independent, but could be useful when considering the Poisson algebra with the involution $\iota_{23}$. In fact, $G_{3}$ satisfies the simple relation

$$
G_{3}+F_{3}=\gamma H-e_{1}^{2} \text {. }
$$

The Poisson algebra for the general case seems to be quite complicated. Restricting the parameters introduces additional isometries. Setting any two of the parameters to zero reduces the metric to a flat or constant curvature case. However,

1. Setting just $\alpha=0$, we have the isometry algebra $\left\langle e_{1}, e_{3}, h_{3}\right\rangle$, satisfying Euclidean relations.

2. Setting just $\beta=0$, we have the isometry algebra $\left\langle e_{1}, e_{2}, h_{2}\right\rangle$, satisfying Euclidean relations. This case is related to that of $\alpha=0$ by the action of the involution $\iota_{23}$.

3. Setting just $\gamma=0$, we have the isometry algebra $\left\langle e_{1}, h_{1}, f_{1}\right\rangle$, satisfying the relations of $\mathfrak{s l}(2)$.

\subsubsection{The Poisson algebra of integrals when $\alpha=0$}

Now we have

$$
\varphi=\frac{q_{2}^{2}}{\beta+\gamma q_{2}^{2}} \quad \text { and } \quad \psi=-2 \beta \frac{q_{1}^{2}+q_{3}^{2}}{q_{2}^{2}}+2 \gamma q_{2}^{2},
$$

so we have lost the involutive symmetry (so no integrals $G_{i}$ ), but gained additional Killing vectors, with isometry algebra $\left\langle e_{1}, e_{3}, h_{3}\right\rangle$, satisfying the Euclidean relations

$$
\left\{e_{1}, e_{3}\right\}=0, \quad\left\{e_{1}, h_{3}\right\}=-2 e_{3}, \quad\left\{e_{3}, h_{3}\right\}=2 e_{1} .
$$

If we define $K_{1}=e_{1}, K_{2}=e_{3}, K_{3}=h_{3}$, then $\left\{K_{i}, F_{j}\right\}$ are easily determined, requiring only the introduction of one additional element $F_{4}=e_{2} h_{4}-\frac{4 \beta q_{3}}{q_{2}^{2}} H$, when calculating $\left\{K_{2}, F_{1}\right\}$ and $\left\{K_{3}, F_{2}\right\}$. The formula for $F_{4}$ is easily determined from Table 1 , by considering $\left\{e_{3}, e_{2} f_{2}\right\}=$ $-e_{2} h_{4}$. The cubic expressions $\left\{F_{i}, F_{j}\right\}$ can all be written in terms of our linear and quadratic functions. The full set of Poisson relations is given in Table 3. Adding $H$, we have an 8dimensional algebra obeying constraints:

$$
\begin{aligned}
& I_{1}=K_{1}^{2}+K_{2}^{2}+F_{3}-\gamma H=0, \quad I_{2}=K_{1} F_{4}-2 K_{2} F_{2}+2 K_{3} F_{3}=0, \\
& I_{3}=4 F_{2}^{2}-8 F_{1} F_{3}+F_{4}^{2}-16 \beta \gamma H^{2}=0 .
\end{aligned}
$$

Remark 4.2. Notice that the first two expressions are related to $\mathcal{C}_{2}^{(h e)}$ and $\mathcal{C}_{4}^{(h e)}$ respectively (see $(2.3 \mathrm{~g}))$. We could, in fact, use $I_{1}=0$ to eliminate $F_{3}$ and just write a $6 \times 6$ table, but $F_{3}$ was an integral part of the definition of this case. 
Table 3. The Poisson algebra of first integrals when $\alpha=0$.

\begin{tabular}{|c||c|c|c||c|c|c|c|}
\hline & $K_{1}$ & $K_{2}$ & $K_{3}$ & $F_{1}$ & $F_{2}$ & $F_{3}$ & $F_{4}$ \\
\hline \hline$K_{1}$ & 0 & 0 & $-2 K_{2}$ & $-2 F_{2}$ & $-2 F_{3}$ & 0 & 0 \\
\hline$K_{2}$ & & 0 & $2 K_{1}$ & $-F_{4}$ & 0 & 0 & $-4 F_{3}$ \\
\hline$K_{3}$ & & & 0 & 0 & $F_{4}$ & 0 & $-4 F_{2}$ \\
\hline \hline$F_{1}$ & & & & 0 & $-2\left(2 K_{1} F_{1}+K_{3} F_{4}\right)$ & $-2\left(2 K_{1} F_{2}+K_{2} F_{4}\right)$ & $8\left(K_{3} F_{2}-K_{2} F_{1}\right)$ \\
\hline$F_{2}$ & & & & & 0 & $-4 K_{1} F_{3}$ & $2\left(2 K_{2} F_{2}+2 K_{3} F_{3}-K_{1} F_{4}\right)$ \\
\hline$F_{3}$ & & & & & & 0 & $8 K_{2} F_{3}$ \\
\hline$F_{4}$ & & & & & & & 0 \\
\hline
\end{tabular}

Table 4. The action of $\iota_{13}$ on $H$ and its integrals.

\begin{tabular}{|l||l||l|l|l||l|l|l|l|}
\hline & $H$ & $K_{1}$ & $K_{2}$ & $K_{3}$ & $F_{1}$ & $F_{2}$ & $F_{3}$ & $F_{4}$ \\
\hline \hline$\iota_{13}$ & $H$ & $K_{2}$ & $K_{1}$ & $-K_{3}$ & $F_{1}$ & $\frac{1}{2} F_{4}$ & $F_{3}$ & $2 F_{2}$ \\
\hline
\end{tabular}

Since $H$ is invariant under the action of $\iota_{13}$, the Poisson algebra has this symmetry. The action of $\iota_{13}$ is summarised in Table 4. Several of the entries in Table 3 are related through this involution. We also have that the constraints (4.5) transform as

$$
\iota_{13}:\left(I_{1}, I_{2}, I_{3}\right) \mapsto\left(I_{1},-I_{2}, I_{3}\right) .
$$

\subsubsection{The Poisson algebra of integrals when $\gamma=0$}

When $\gamma=0$, the Hamiltonian $H$ is invariant under the action of $\iota_{e f}$ (as is the 3 -dimensional isometry algebra). We define $K_{1}=e_{1}, K_{2}=h_{1}, K_{3}=f_{1}$, with $F_{1}, F_{2}, F_{3}$ given by (4.2). The action of $\iota_{e f}$ on $F_{2}$ and $F_{3}$ gives us two more quadratic elements, which close the algebra:

$$
F_{4}=f_{2} h_{2}-\frac{4 \beta q_{1}\left(q_{1}^{2}+q_{2}^{2}+q_{3}^{2}\right)}{q_{2}^{2}} H, \quad F_{5}=f_{2}^{2}-\frac{4 \beta\left(q_{1}^{2}+q_{2}^{2}+q_{3}^{2}\right)^{2}}{q_{2}^{2}} H .
$$

The action of $\iota_{e f}$ is summarised in Table 5. A consequence of this is that we can deduce the entries for $\left\{K_{i}, F_{4}\right\}$ and $\left\{K_{i}, F_{5}\right\}$ (in Table 6) from those of $\left\{K_{j}, F_{2}\right\}$ and $\left\{K_{j}, F_{3}\right\}$. The entries $\left\{F_{3}, F_{4}\right\}$ and $\left\{F_{2}, F_{5}\right\}$ are similarly related. Some of the longer entries are labelled $P_{i j}$ and listed below.

The longer entries are given by

$$
\begin{aligned}
& P_{24}=8 K_{2}\left(-F_{1}-2 \mathcal{C}_{K}+(2 \alpha+\beta) H\right), \quad P_{25}=-4\left(K_{2} F_{4}+2 K_{3} F_{1}+4 \beta K_{3} H\right), \\
& P_{34}=-4\left(K_{2} F_{2}-K_{1} F_{1}-2 \beta K_{1} H\right), \quad P_{35}=-8 K_{2}\left(F_{1}+2 \beta H\right),
\end{aligned}
$$

where $\mathcal{C}_{K}=K_{1} K_{3}+\frac{1}{4} K_{2}^{2}$ is the Casimir function of the isometry algebra.

Adding $H$, we have a 9-dimensional algebra obeying the constraints:

$$
\begin{aligned}
& I_{1}=K_{1} F_{1}+K_{2} F_{2}-2 K_{3} F_{3}-2 \beta K_{1} H=0, \\
& I_{2}=K_{1} F_{5}+K_{2} F_{4}-2 K_{3} F_{1}+4 \beta K_{3} H=0, \\
& I_{3}=F_{1} F_{2}-F_{3} F_{4}+2 K_{2}\left(K_{1} F_{1}+K_{2} F_{2}-2 K_{3} F_{3}\right)-2 \beta H F_{2}=0, \\
& I_{4}=F_{1} F_{4}-F_{2} F_{5}+2 K_{2}\left(K_{1} F_{5}+K_{2} F_{4}-2 K_{3} F_{1}\right)-2 \beta H F_{4}=0 .
\end{aligned}
$$


Table 5. The action of $\iota_{e f}$ on $H$ and its integrals.

\begin{tabular}{|c||c||c|c|c||c|c|c||c|c|}
\hline & $H$ & $K_{1}$ & $K_{2}$ & $K_{3}$ & $F_{1}$ & $F_{2}$ & $F_{3}$ & $F_{4}$ & $F_{5}$ \\
\hline \hline$\iota_{e f}$ & $H$ & $-K_{3}$ & $-K_{2}$ & $-K_{1}$ & $F_{1}$ & $-\frac{1}{2} F_{4}$ & $\frac{1}{4} F_{5}$ & $-2 F_{2}$ & $4 F_{3}$ \\
\hline
\end{tabular}

Table 6. The Poisson algebra of first integrals when $\gamma=0$.

\begin{tabular}{|c||c|c|c||c|c|c||c|c|}
\hline & $K_{1}$ & $K_{2}$ & $K_{3}$ & $F_{1}$ & $F_{2}$ & $F_{3}$ & $F_{4}$ & $F_{5}$ \\
\hline \hline$K_{1}$ & 0 & $2 K_{1}$ & $-K_{2}$ & $-2 F_{2}$ & $-2 F_{3}$ & 0 & $-6 F_{1}-8 \mathcal{C}_{K}+4(2 \alpha+\beta) H$ & $-4 F_{4}$ \\
\hline$K_{2}$ & & 0 & $2 K_{3}$ & 0 & $-2 F_{2}$ & $-4 F_{3}$ & $2 F_{4}$ & $4 F_{5}$ \\
\hline$K_{3}$ & & & 0 & $-F_{4}$ & $-3 F_{1}-4 \mathcal{C}_{K}+2(2 \alpha+\beta) H$ & $-2 F_{2}$ & $-F_{5}$ & 0 \\
\hline \hline$F_{1}$ & & & & 0 & $8\left(K_{3} F_{3}+\beta K_{1} H\right)$ & $4 K_{2} F_{3}$ & $4\left(K_{1} F_{5}+4 \beta K_{3} H\right)$ & $-4 K_{2} F_{5}$ \\
\hline$F_{2}$ & & & & & 0 & $-4 K_{1} F_{3}$ & $P_{24}$ & $P_{25}$ \\
\hline$F_{3}$ & & & & & & 0 & $P_{34}$ & $P_{35}$ \\
\hline \hline$F_{4}$ & & & & & & & & \\
\hline$F_{5}$ & & & & & & & & $-8 K_{3} F_{5}$ \\
\hline
\end{tabular}

Remark 4.3. Notice that the first two expressions are related to the Casimir $\mathcal{C}_{4}^{(0)}$, of the algebra $\mathfrak{g}^{(0)}$ of Section 2.1. Under the action of $\iota_{e f}$, they obey

$$
\left(I_{1}, I_{2}, I_{3}, I_{4}\right) \mapsto\left(\frac{1}{2} I_{2}, 2 I_{1},-\frac{1}{2} I_{4},-2 I_{3}\right) .
$$

\section{$4.2 \quad F_{1}=e_{1} h_{2}+\psi_{1}\left(q_{1}, q_{2}, q_{3}\right) H, G_{1}=e_{1} h_{3}+\psi_{2}\left(q_{1}, q_{2}, q_{3}\right) H$}

We again take the Hamiltonian (4.1), with first integral $K=e_{1}$, and consider the chains of functions generated by

$$
\begin{array}{ll}
F_{1}=e_{1} h_{2}+\psi_{1}\left(q_{1}, q_{2}, q_{3}\right) H, & F_{2}=\frac{1}{2}\left\{F_{1}, e_{1}\right\}=e_{1} e_{2}+\frac{1}{2} \psi_{1 q_{1}} H, \\
G_{1}=e_{1} h_{3}+\psi_{2}\left(q_{1}, q_{2}, q_{3}\right) H, & G_{2}=\frac{1}{2}\left\{G_{1}, e_{1}\right\}=e_{1} e_{3}+\frac{1}{2} \psi_{2 q_{1}} H,
\end{array}
$$

related through the involution $\iota_{23}$. The conditions $\left\{F_{1}, H\right\}=0$ and $\left\{G_{1}, H\right\}=0$ give the functions

$$
\varphi=\frac{1}{\alpha q_{2}+\beta q_{3}+\gamma}, \quad \psi_{1}=-\frac{\alpha}{2} q_{1}^{2}, \quad \psi_{2}=-\frac{\beta}{2} q_{1}^{2},
$$

so $\left\{F_{2}, e_{1}\right\}=-\frac{\alpha}{2} H$ and $\left\{G_{2}, e_{1}\right\}=-\frac{\beta}{2} H$. The integrals $H, F_{1}, F_{2}, G_{1}, G_{2}$ are functionally independent.

Remark 4.4 (the Stäckel transform). Again, the Hamiltonian (4.1), but with $\varphi$ given by (4.7c) can be regarded as the Stäckel transform of the Euclidean kinetic energy with potential $V=\varphi^{-1}$. In the classification of [4], this example corresponds to a reduction of $V_{[0]}$, given in [4, Section 5], by equating $\left(q_{1}, q_{2}, q_{3}\right)=(z, x, y)$ and $(\alpha, \beta, \gamma)=\left(a_{1}, a_{2}, a_{5}\right)$.

This solution has a 3-dimensional isometry algebra

$$
K_{1}=e_{1}, \quad K_{2}=\beta e_{2}-\alpha e_{3}, \quad K_{3}=\beta h_{2}-\alpha h_{3},
$$

satisfying

$$
\left\{K_{1}, K_{2}\right\}=0, \quad\left\{K_{1}, K_{3}\right\}=-2 K_{2}, \quad\left\{K_{2}, K_{3}\right\}=2\left(\alpha^{2}+\beta^{2}\right) K_{1},
$$


Table 7. The Poisson algebra of first integrals of (4.1), with (4.7c).

\begin{tabular}{|c||c|c|c||c|c|c|c|c|c|}
\hline & $K_{1}$ & $K_{2}$ & $K_{3}$ & $F_{1}$ & $F_{2}$ & $F_{3}$ & $F_{4}$ & $F_{5}$ & $F_{6}$ \\
\hline \hline$K_{1}$ & 0 & 0 & $-2 K_{2}$ & $-2 F_{2}$ & $\frac{1}{2} \alpha H$ & $-2 F_{4}$ & 0 & 0 & 0 \\
\hline$K_{2}$ & & 0 & $2 \delta K_{1}$ & $2 \beta K_{1}^{2}$ & 0 & $-4 \delta F_{2}+8 \beta K_{1} K_{2}$ & $\alpha \delta H$ & $-\alpha \beta H$ & $\bar{\alpha}\left(\delta F_{4}-2 \beta K_{2}^{2}\right)$ \\
\hline$K_{3}$ & & 0 & $F_{3}$ & $F_{4}-2 \beta K_{1}^{2}$ & $4 \beta K_{1} K_{3}-8 \delta F_{1}-4 \alpha F_{6}$ & $-4 \delta F_{2}-4 \beta K_{1} K_{2}$ & $4\left(\beta F_{2}+K_{1} K_{2}\right)$ & $\bar{\alpha}\left(\delta F_{3}-2 \beta K_{2} K_{3}\right)$ \\
\hline \hline$F_{1}$ & & & 0 & $-2 K_{1} F_{5}$ & $4 K_{3} F_{5}-8 \beta K_{1} F_{1}$ & $-4 K_{1}\left(K_{1} K_{2}+\beta F_{2}\right)$ & $8 K_{1} F_{2}$ & $\bar{\alpha} K_{1}\left(\beta F_{3}-2 K_{2} K_{3}\right)$ \\
\hline$F_{2}$ & & & & 0 & $4\left(K_{1}^{2} K_{2}-\beta K_{1} F_{2}+K_{2} F_{5}\right)$ & $\alpha \beta K_{1} H$ & $-2 \alpha K_{1} H$ & $\bar{\alpha} K_{1}\left(\beta F_{4}-2 K_{2}^{2}\right)$ \\
\hline$F_{3}$ & & & & & 0 & $P_{34}$ & $P_{35}$ & $P_{36}$ \\
\hline$F_{4}$ & & & & & & 0 & $-2 \alpha K_{2} H$ & $4 \bar{\alpha} K_{2}\left(\delta\left(K_{1}^{2}-F_{5}\right)-K_{2}^{2}\right)$ \\
\hline$F_{5}$ & & & & & & & 0 & $2 \bar{\alpha} K_{2}\left(F_{4}+2 \beta F_{5}-2 \beta K_{1}^{2}\right)$ \\
\hline$F_{6}$ & & & & & & & & 0 \\
\hline
\end{tabular}

with Casimir

$$
\mathcal{C}_{K}=\left(\alpha^{2}+\beta^{2}\right) K_{1}^{2}+K_{2}^{2}
$$

We then find that

$$
\alpha G_{1}-\beta F_{1}+K_{1} K_{3}=0, \quad \text { and } \quad \alpha G_{2}-\beta F_{2}+K_{1} K_{2}=0,
$$

with the integrals $H, K_{1}, K_{2}, K_{3}, F_{1}$ being functionally independent.

We can use $\left\langle K_{1}, K_{2}, K_{3}, F_{1}, F_{2}\right\rangle$ to generate a Poisson algebra. The action of $K_{i}$ on $F_{i}$ and the Poisson bracket $\left\{F_{1}, F_{2}\right\}$ require the introduction of four more quadratic elements:

$$
\begin{aligned}
& F_{3}=2 K_{2} h_{2}-\alpha K_{1} h_{4}-2 \alpha q_{1}\left(\beta q_{2}-\alpha q_{3}\right) H, \quad F_{4}=2 e_{2} K_{2}-\alpha\left(\beta q_{2}-\alpha q_{3}\right) H, \\
& F_{5}=e_{1}^{2}-e_{2}^{2}+\alpha q_{2} H, \quad F_{6}=K_{2} h_{4}+\left(\beta q_{2}-\alpha q_{3}\right)^{2} H,
\end{aligned}
$$

satisfying the relations given in Table 7 . In this table we have $\delta=\alpha^{2}+\beta^{2}, \bar{\alpha}=\frac{2}{\alpha}$ and

$$
\begin{aligned}
& P_{34}=4 K_{1}\left(\beta F_{4}-2 \alpha^{2} K_{1}^{2}-6 K_{2}^{2}+2 \alpha^{2} \gamma H\right), \\
& P_{35}=8 K_{1}\left(3 F_{4}+\beta F_{5}-\beta K_{1}^{2}\right)-24 K_{2} F_{2}-8 \alpha K_{3} H, \\
& P_{36}=\frac{4}{\alpha} K_{3}\left(\beta F_{4}-2 \alpha^{2} K_{1}^{2}-4 K_{2}^{2}+2 \alpha^{2} \gamma H\right)+\frac{4 \beta}{\alpha} K_{2} F_{3} .
\end{aligned}
$$

This 10-dimensional algebra (including $H$ itself) is constrained by the following relations

$$
\begin{aligned}
& I_{1}=K_{1} F_{3}+2 K_{3} F_{2}-4 K_{2} F_{1}=0, \quad I_{2}=2 \alpha K_{1} F_{6}+K_{2} F_{3}-K_{3} F_{4}=0, \\
& I_{3}=\alpha K_{3} H+4 K_{2} F_{2}-2 K_{1} F_{4}=0, \quad I_{4}=8 K_{1} K_{2} F_{5}-8 K_{1}^{3} K_{2}+4 F_{2} F_{4}+\alpha F_{3} H=0, \\
& I_{5}=\left(2 \alpha^{2}+\beta^{2}\right) K_{1}^{2}+K_{2}^{2}-\left(\alpha^{2}+\beta^{2}\right) F_{5}-\beta F_{4}-\alpha^{2} \gamma H=0 .
\end{aligned}
$$

\section{Systems with $K=h_{1}$}

In the case $K=h_{1}$, we consider the Hamiltonian (2.1) of the form

$$
H=\varphi\left(q_{1}, q_{2}, q_{3}\right)\left(p_{1}^{2}+p_{2}^{2}+p_{3}^{2}\right)=q_{1}^{2} \Phi\left(\frac{q_{2}}{q_{1}}, \frac{q_{3}}{q_{1}}\right)\left(p_{1}^{2}+p_{2}^{2}+p_{3}^{2}\right),
$$

which is the most general form commuting with $h_{1}$. This is also generally invariant with respect to the involution $\iota_{e f}$, so we choose our functions $F_{i}$ to have this invariance, noting that $\iota_{e f}:\left(e_{2}, h_{2}, f_{2}\right) \mapsto\left(-\frac{1}{2} f_{2}, h_{2},-2 e_{2}\right)$. 


\section{$5.1 \quad F_{1}=e_{2} h_{2}+\psi_{1}\left(q_{1}, q_{2}, q_{3}\right) H, F_{2}=f_{2} h_{2}+\psi_{2}\left(q_{1}, q_{2}, q_{3}\right) H$, $F_{3}=h_{2}^{2}+\psi_{3}\left(q_{1}, q_{2}, q_{3}\right) H$}

With the Hamiltonian (5.1), with first integral $K=h_{1}$, we consider functions of the form

$$
\begin{aligned}
& F_{1}=e_{2} h_{2}+\psi_{1}\left(q_{1}, q_{2}, q_{3}\right) H, \quad F_{2}=f_{2} h_{2}+\psi_{2}\left(q_{1}, q_{2}, q_{3}\right) H, \\
& F_{3}=h_{2}^{2}+\psi_{3}\left(q_{1}, q_{2}, q_{3}\right) H,
\end{aligned}
$$

and require the conditions $\left\{F_{i}, H\right\}=0$ to find

$$
\begin{aligned}
& \varphi=\frac{q_{2}^{2} q_{3}^{2} \sqrt{q_{1}^{2}+q_{2}^{2}}}{\left(\alpha q_{2}^{2}+\beta q_{3}^{2}\right) \sqrt{q_{1}^{2}+q_{2}^{2}}+\gamma q_{1} q_{3}^{2}}, \quad \psi_{1}=-\frac{\gamma\left(2 q_{1}^{2}+q_{2}^{2}\right)+2 \beta q_{1} \sqrt{q_{1}^{2}+q_{2}^{2}}}{q_{2}^{2} \sqrt{q_{1}^{2}+q_{2}^{2}}}, \\
& \psi_{2}=2\left(q_{1}^{2}+q_{2}^{2}+q_{3}^{2}\right) \psi_{1}, \quad \psi_{3}=-\frac{4 q_{1}\left(\beta q_{1}+\gamma \sqrt{q_{1}^{2}+q_{2}^{2}}\right)}{q_{2}^{2}} .
\end{aligned}
$$

If we extend $\iota_{e f}$ to act on the parameters: $(\alpha, \beta, \gamma) \mapsto(\alpha, \beta,-\gamma)$ then

$$
\left(H, F_{1}, F_{2}, F_{3}\right) \mapsto\left(H,-\frac{1}{2} F_{2},-2 F_{1}, F_{3}\right) .
$$

Remark 5.1 (the Stäckel transform). Again, the Hamiltonian (5.1), with $\varphi$ given by (5.2) can be regarded as the Stäckel transform of the Euclidean kinetic energy with potential $V=\varphi^{-1}$. In the classification of [4], this example corresponds to a reduction of system iii, given in [4, Section 7], by equating $\left(q_{1}, q_{2}, q_{3}\right)=(z, y, x)$ and $(\alpha, \beta, \gamma)=\left(a_{3}, a_{4}, a_{5}\right)$.

The integrals $H, K, F_{1}, F_{2}, F_{3}$ are functionally independent. The simpler Poisson relations are

$$
\begin{aligned}
& \left\{F_{1}, K\right\}=2 F_{1}, \quad\left\{F_{2}, K\right\}=-2 F_{2}, \quad\left\{F_{3}, K\right\}=0, \\
& \left\{F_{1}, F_{2}\right\}=4 K\left(2 \beta H-F_{3}\right) .
\end{aligned}
$$

Again, we can restrict the parameters to increase the algebra of isometries:

1. By setting $\alpha=0$, we have the isometry algebra $\left\langle e_{3}, h_{1}, f_{3}\right\rangle$, satisfying the relations of $\mathfrak{s l}(2)$.

2. By setting $\beta=0$, we just have the single isometry $h_{1}$.

3. By setting $\gamma=0$, the metric reduces to that of Section 4.1 (with $\gamma=0$ ) so has the isometry algebra $\left\langle e_{1}, h_{1}, f_{1}\right\rangle$.

\subsubsection{The Poisson algebra of integrals when $\alpha=0$}

Now we have

$$
\varphi=\frac{q_{2}^{2} \sqrt{q_{1}^{2}+q_{2}^{2}}}{\beta \sqrt{q_{1}^{2}+q_{2}^{2}}+\gamma q_{1}},
$$

with $\psi_{i}$ as before, and gain additional Killing vectors, with isometry algebra $\left\langle h_{1}, e_{3}, f_{3}\right\rangle$.

If we define $K_{1}=h_{1}, K_{2}=e_{3}, K_{3}=f_{3}$, then $\left\{K_{i}, F_{j}\right\}$ are easily determined, requiring only the introduction of one additional element $F_{4}=-h_{2} h_{4}-4 q_{3} \psi_{1} H$, when calculating $\left\{K_{2}, F_{2}\right\}$ and $\left\{K_{3}, F_{1}\right\}$. The formula for $F_{4}$ is easily determined from Table 1 , by considering $\left\{e_{3}, f_{2} h_{2}\right\}=$ $-h_{2} h_{4}$. The cubic expressions $\left\{F_{i}, F_{j}\right\}$ can all be written in terms of our linear and quadratic functions. The full set of Poisson relations is given in Table 8. Since $H$ is invariant under the action of $\iota_{e f}$, the Poisson algebra has this symmetry. The action of $\iota_{e f}$ is summarised in Table 9. Several of the entries in Table 8 are related through this involution.

Adding $H$, we have an 8-dimensional algebra obeying the constraints:

$$
I_{1}=K_{1}^{2}+2 K_{2} K_{3}+F_{3}-4 \beta H=0, \quad I_{2}=K_{1} F_{4}-2 K_{2} F_{2}+2 K_{3} F_{1}=0 .
$$

Remark 5.2. Both of these are Casimirs of the algebra, with the first extending the Casimir of the isometry algebra and the second being related to $\mathcal{C}_{4}^{(13)}$ of $(2.3 \mathrm{c})$. 
Table 8. The Poisson algebra of first integrals when $\alpha=0$.

\begin{tabular}{|c||c|c|c||c|c|c|c|}
\hline & $K_{1}$ & $K_{2}$ & $K_{3}$ & $F_{1}$ & $F_{2}$ & $F_{3}$ & $F_{4}$ \\
\hline \hline$K_{1}$ & 0 & $-2 K_{2}$ & $2 K_{3}$ & $-2 F_{1}$ & $2 F_{2}$ & 0 & 0 \\
\hline$K_{2}$ & & 0 & $-2 K_{1}$ & 0 & $F_{4}$ & 0 & $4 F_{1}$ \\
\hline$K_{3}$ & & & 0 & $F_{4}$ & 0 & 0 & $4 F_{2}$ \\
\hline \hline$F_{1}$ & & & & 0 & $4 K_{1}\left(2 \beta H-F_{3}\right)$ & $2\left(K_{2} F_{4}-2 K_{1} F_{1}\right)$ & $8 K_{2}\left(2 \beta H-F_{3}\right)$ \\
\hline$F_{2}$ & & & & & 0 & $2\left(K_{3} F_{4}+2 K_{1} F_{2}\right)$ & $8 K_{3}\left(2 \beta H-F_{3}\right)$ \\
\hline$F_{3}$ & & & & & & 0 & $-8\left(K_{2} F_{2}+K_{3} F_{1}\right)$ \\
\hline$F_{4}$ & & & & & & & 0 \\
\hline
\end{tabular}

Table 9. The action of $\iota_{e f}$ on $H$ and its integrals.

\begin{tabular}{|c||c||c|c|c||c|c|c||c|}
\hline & $H$ & $K_{1}$ & $K_{2}$ & $K_{3}$ & $F_{1}$ & $F_{2}$ & $F_{3}$ & $F_{4}$ \\
\hline \hline$\iota_{e f}$ & $H$ & $-K_{1}$ & $-\frac{1}{2} K_{3}$ & $-2 K_{2}$ & $-\frac{1}{2} F_{2}$ & $-2 F_{1}$ & $F_{3}$ & $F_{4}$ \\
\hline
\end{tabular}

\section{Systems with $K=h_{4}$}

In the case $K=h_{4}$, we consider the Hamiltonian (2.1) of the form

$$
H=\varphi\left(q_{1}, q_{2}, q_{3}\right)\left(p_{1}^{2}+p_{2}^{2}+p_{3}^{2}\right)=\Phi\left(q_{1}, q_{2}^{2}+q_{3}^{2}\right)\left(p_{1}^{2}+p_{2}^{2}+p_{3}^{2}\right),
$$

which is the most general form commuting with $h_{4}$. This is also generally invariant with respect to the involution $\iota_{23}$, so we choose our functions $F_{i}$ to have this invariance.

\section{$6.1 \quad F_{1}=e_{1}^{2}+\psi_{1}\left(q_{1}, q_{2}, q_{3}\right) H, F_{2}=e_{1} e_{2}+\psi_{2}\left(q_{1}, q_{2}, q_{3}\right) H$, $F_{3}=e_{1} e_{3}+\psi_{3}\left(q_{1}, q_{2}, q_{3}\right) H$}

With the Hamiltonian (6.1), with first integral $K=h_{4}$, we consider functions of the form

$$
\begin{aligned}
& F_{1}=e_{1}^{2}+\psi_{1}\left(q_{1}, q_{2}, q_{3}\right) H, \quad F_{2}=e_{1} e_{2}+\psi_{2}\left(q_{1}, q_{2}, q_{3}\right) H, \\
& F_{3}=e_{1} e_{3}+\psi_{3}\left(q_{1}, q_{2}, q_{3}\right) H
\end{aligned}
$$

and require the conditions $\left\{F_{i}, H\right\}=0$ to find

$$
\begin{aligned}
& H=\varphi\left(q_{1}, q_{2}, q_{3}\right)\left(p_{1}^{2}+p_{2}^{2}+p_{3}^{2}\right)=\frac{1}{\alpha\left(q_{1}^{2}+q_{2}^{2}+q_{3}^{2}\right)+\beta q_{1}+\gamma}\left(p_{1}^{2}+p_{2}^{2}+p_{3}^{2}\right), \\
& \psi_{1}=-\alpha q_{1}^{2}-\beta q_{1}, \quad \psi_{2}=-\left(\alpha q_{1}+\frac{\beta}{2}\right) q_{2}, \quad \psi_{3}=-\left(\alpha q_{1}+\frac{\beta}{2}\right) q_{3},
\end{aligned}
$$

which clearly transform as $\left(H, F_{1}, F_{2}, F_{3}\right) \mapsto\left(H, F_{1}, F_{3}, F_{2}\right)$ under $\iota_{23}$.

Remark 6.1 (the Stäckel transform). The Hamiltonian (6.2), can be regarded as the Stäckel transform of the Euclidean kinetic energy with potential $V=\varphi^{-1}$. In the classification of [4], this example corresponds to a reduction of $V_{[0]}$, given in [4, Section 5], by equating $\left(q_{1}, q_{2}, q_{3}\right)=$ $(x, y, z)$ and $(\alpha, \beta, \gamma)=\left(a_{4}, a_{1}, a_{5}\right)$.

This $H$ has first order symmetries

$$
K_{1}=h_{4}, \quad K_{2}=\alpha h_{2}+\beta e_{2}, \quad K_{3}=\alpha h_{3}+\beta e_{3},
$$


Table 10. The Poisson algebra of first integrals of (6.2).

\begin{tabular}{|c|c|c|c|c|c|c|c|c|c|}
\hline & $\overline{K_{1}}$ & $K_{2}$ & $K_{3}$ & $F_{1}$ & $\overline{F_{2}}$ & $F_{3}$ & $F_{4}$ & $F_{5}$ & $F_{6}$ \\
\hline$K_{1}$ & 0 & $-4 K_{3}$ & $4 K_{2}$ & 0 & $-4 F_{3}$ & $4 F_{2}$ & $-8 F_{5}$ & $4\left(F_{4}-F_{6}\right)$ & $8 F_{5}$ \\
\hline$K_{2}$ & & 0 & $-\alpha^{2} K_{1}$ & $4 \alpha F_{2}$ & $2 \alpha\left(F_{4}-F_{1}\right)+\frac{1}{2} \beta^{2} H$ & $2 \alpha F_{5}$ & $-4 \alpha F_{2}$ & $-2 \alpha F_{3}$ & 0 \\
\hline$K_{3}$ & & & 0 & $4 \alpha F_{3}$ & $2 \alpha F_{5}$ & $2 \alpha\left(F_{6}-F_{1}\right)+\frac{1}{2} \beta^{2} H$ & 0 & $-2 \alpha F_{2}$ & $-4 \alpha F_{3}$ \\
\hline$F_{1}$ & & & & 0 & $-K_{2} H$ & $-K_{3} H$ & 0 & 0 & 0 \\
\hline$F_{2}$ & & & & & 0 & $\frac{1}{4} \alpha K_{1} H$ & $-K_{2} H$ & $-\frac{1}{2} K_{3} H$ & 0 \\
\hline$F_{3}$ & & & & & & 0 & 0 & $-\frac{1}{2} K_{2} H$ & $-K_{3} H$ \\
\hline$F_{4}$ & & & & & & & 0 & $\frac{1}{2} \alpha K_{1} H$ & 0 \\
\hline$F_{5}$ & & & & & & & & 0 & $\frac{1}{2} \alpha K_{1} H$ \\
\hline$F_{6}$ & & & & & & & & & 0 \\
\hline
\end{tabular}

Table 11. The action of $\iota_{23}$ on $H$ and its integrals.

\begin{tabular}{|l||c||c|c|c||c|c|c||c|c|c|}
\hline & $H$ & $K_{1}$ & $K_{2}$ & $K_{3}$ & $F_{1}$ & $F_{2}$ & $F_{3}$ & $F_{4}$ & $F_{5}$ & $F_{6}$ \\
\hline \hline$\iota_{23}$ & $H$ & $-K_{1}$ & $K_{3}$ & $K_{2}$ & $F_{1}$ & $F_{3}$ & $F_{2}$ & $F_{6}$ & $F_{5}$ & $F_{4}$ \\
\hline
\end{tabular}

satisfying

$$
\left\{K_{1}, K_{2}\right\}=-4 K_{3}, \quad\left\{K_{1}, K_{3}\right\}=4 K_{2}, \quad\left\{K_{3}, K_{2}\right\}=\alpha^{2} K_{1} .
$$

The action of $K_{i}$ on $F_{2}$ and $F_{3}$ introduces three new quadratic elements to our algebra:

$$
F_{4}=e_{2}^{2}-\alpha q_{2}^{2} H, \quad F_{5}=e_{2} e_{3}-\alpha q_{2} q_{3} H, \quad F_{6}=e_{3}^{2}-\alpha q_{3}^{2} H,
$$

giving 9 first integrals, which satisfies the Poisson relations of Table 10. Adding $H$ gives us a 10-dimensional algebra obeying the constraints:

$$
\begin{aligned}
& I_{1}=\alpha K_{1} F_{2}-2 K_{2} F_{5}+2 K_{3} F_{4}=0, \quad I_{2}=\alpha K_{1} F_{3}-2 K_{2} F_{6}+2 K_{3} F_{5}=0, \\
& I_{3}=4 \alpha K_{1} F_{1}-\beta^{2} K_{1} H-8 K_{2} F_{3}+8 K_{3} F_{2}=0, \\
& I_{4}=8 F_{3} F_{4}-8 F_{2} F_{5}+K_{1} K_{2} H=0, \quad I_{5}=8 F_{3} F_{5}-8 F_{2} F_{6}+K_{1} K_{3} H=0 .
\end{aligned}
$$

Remark 6.2. The first three of these are related to the Casimir $\mathcal{C}_{4}^{(h e)}$ of (2.3g). The elements $K_{i}$ and $F_{i}$ are built from the algebra $\mathfrak{g}^{(h e)}$ of Section 2.1. We also have a linear relation $F_{1}+F_{4}+F_{6}=$ $\gamma H$, which could be used to eliminate $F_{6}$, but this would complicate Table 11.

The Hamiltonian (6.2) is invariant under the action of $\iota_{23}$, as is this algebra (see Table 11). Several of the entries of Table 10 (such as $\left\{K_{2}, F_{2}\right\}$ and $\left\{K_{3}, F_{3}\right\}$ ) are related through this involution.

\section{$7 \quad$ Reductions of the system of Section 4.1}

We now consider reductions of the Hamiltonian

$$
H=\frac{q_{2}^{2} q_{3}^{2}}{\alpha q_{2}^{2}+\beta q_{3}^{2}+\gamma q_{2}^{2} q_{3}^{2}}\left(p_{1}^{2}+p_{2}^{2}+p_{3}^{2}\right),
$$


associated with the restrictions of Sections 4.1.1 and 4.1.2. We adapt coordinates to the appropriate invariant, rendering it as $P_{3}$. The 2-dimensional reduction is then $P_{3}=\mu$, but we keep the label $P_{3}$ so that we can later transform (in the 3-dimensional context) between these reductions.

In this first section on reductions we give more detailed calculations. The same methods are used in later sections, but we omit the details.

\subsection{Reductions when $\alpha=0$}

For the case with (4.4a) and isometry algebra (4.4b) we use respectively $K_{2}=e_{3}$ and $K_{3}=h_{3}$ to reduce to 2 dimensions.

\subsubsection{Reduction using the isometry $K_{2}=e_{3}$}

Our original coordinate system $\left(q_{1}, q_{2}, q_{3}\right)$ is already adapted to $e_{3}=p_{3}=P_{3}$. Since $\left\{F_{2}, e_{3}\right\}=$ $\left\{F_{3}, e_{3}\right\}=0$, we can reduce these integrals in this case, with

$$
H=\frac{q_{2}^{2}}{\beta+\gamma q_{2}^{2}}\left(p_{1}^{2}+p_{2}^{2}\right)+\frac{q_{2}^{2} p_{3}^{2}}{\beta+\gamma q_{2}^{2}}, \quad F_{2}=e_{2} h_{2}-\frac{2 \beta q_{1}}{q_{2}^{2}} H, \quad F_{3}=e_{2}^{2}-\frac{\beta}{q_{2}^{2}} H,
$$

still satisfying $\left\{H, e_{1}\right\}=0,\left\{F_{2}, e_{1}\right\}=2 F_{3},\left\{F_{3}, e_{1}\right\}=0$. The system (7.1) is just the DarbouxKoenigs system of type $D_{2}$ with the potential of "Type D" in [9].

By considering linear and quadratic conformal elements (in 3D) that Poisson commute with $e_{3}$, we can reduce the 10-dimensional conformal algebra to the required 6-dimensional algebra. For example

$$
e_{1}, e_{2}, h_{2}, e_{1} h_{1}-e_{3} h_{3}, h_{3}^{2}-4 e_{1} f_{1}, \text { and } h_{4}^{2}-8 e_{2} f_{2}
$$

give us the standard 6-dimensional subalgebra of conformal symmetries of $2 \mathrm{D}$ space:

$$
\begin{aligned}
& T_{1}=p_{1}, \quad T_{2}=p_{2}, \quad T_{3}=q_{2} p_{1}-q_{1} p_{2}, \quad T_{4}=q_{1} p_{1}+q_{2} p_{2}, \\
& T_{5}=\left(q_{1}^{2}-q_{2}^{2}\right) p_{1}+2 q_{1} q_{2} p_{2}, \quad T_{6}=2 q_{1} q_{2} p_{1}+\left(q_{2}^{2}-q_{1}^{2}\right) p_{2} .
\end{aligned}
$$

Remark 7.1 (the reduction of quadratic elements). In the case of quadratic expressions we set $p_{3}=0$, with only the parts involving $p_{1}^{2}, p_{1} p_{2}, p_{2}^{2}$ representing a quadratic conformal element of the 2-dimensional kinetic energy. From the 3 linear and 3 quadratic expressions, we can deduce 6 linear elements. For example,

$$
e_{1}=p_{1}, \quad e_{1} h_{1}-e_{3} h_{3}=-2 p_{1}\left(q_{1} p_{1}+q_{2} p_{2}\right)-2 q_{1} p_{3}^{2}
$$

give us $T_{1}$ and $T_{4}$.

The functions $F_{2}$ and $F_{3}$ in (7.1) can be written in terms of these, with $T_{1}$ corresponding to the single Killing vector:

$$
F_{2}=-2 T_{2} T_{3}-2 \beta \frac{q_{1}}{q_{2}^{2}} H, \quad F_{3}=T_{2}^{2}-\frac{\beta}{q_{2}^{2}} H
$$

satisfying

$$
\left\{T_{1}, F_{2}\right\}=-2 F_{3}, \quad\left\{T_{1}, F_{3}\right\}=0, \quad\left\{F_{2}, F_{3}\right\}=4 T_{1}\left(T_{1}^{2}+\mu^{2}-\gamma H\right) .
$$




\subsubsection{Reduction using the isometry $K_{3}=h_{3}$}

We adapt coordinates to $h_{3}$ :

$$
Q_{1}=\sqrt{q_{1}^{2}+q_{3}^{2}}, \quad Q_{2}=q_{2}, \quad Q_{3}=-\frac{1}{2} \arctan \left(\frac{q_{1}}{q_{3}}\right) \quad \Rightarrow \quad h_{3}=P_{3},
$$

and

$$
H=\frac{Q_{2}^{2}}{\beta+\gamma Q_{2}^{2}}\left(P_{1}^{2}+P_{2}^{2}+\frac{P_{3}^{2}}{4 Q_{1}^{2}}\right),
$$

which is just the Darboux-Koenigs system of type $D_{2}$ with the potential of "Type B" (parameter $\left.b_{3}\right)$ in [9].

By considering linear and quadratic conformal elements (in 3D) that Poisson commute with $h_{3}$, we can reduce the 10-dimensional conformal algebra to the required 6 -dimensional algebra. For example

$$
h_{1}, e_{2}, f_{2}, e_{1}^{2}+e_{3}^{2}, 4 f_{1}^{2}+f_{3}^{2} \text {, and } 2 e_{1} h_{2}+e_{3} h_{4},
$$

give us (setting $P_{3}=0$ ) the standard conformal algebra for 2D space:

$$
\begin{aligned}
& T_{1}=P_{1}, \quad T_{2}=P_{2}, \quad T_{3}=Q_{2} P_{1}-Q_{1} P_{2}, \quad T_{4}=Q_{1} P_{1}+Q_{2} P_{2}, \\
& T_{5}=\left(Q_{1}^{2}-Q_{2}^{2}\right) P_{1}+2 Q_{1} Q_{2} P_{2}, \quad T_{6}=2 Q_{1} Q_{2} P_{1}+\left(Q_{2}^{2}-Q_{1}^{2}\right) P_{2} .
\end{aligned}
$$

Furthermore, since $e_{1}$ and $e_{3}$ are first integrals, so is

$$
J_{2}=e_{1}^{2}+e_{3}^{2}=T_{1}^{2}+\frac{P_{3}^{2}}{4 Q_{1}^{2}} .
$$

The other independent quadratic integral is $F_{1}$, which, in these coordinates, takes the form

$$
J_{1}=-2 T_{1} T_{5}+\frac{Q_{2}^{2}-Q_{1}^{2}}{2 Q_{1}^{2}} P_{3}^{2}-2\left(\beta-\gamma Q_{1}^{2}\right) H .
$$

From the definition of the constraint $I_{1}$ in (4.5), the integral $F_{3}=-K_{1}^{2}-K_{2}^{2}+\gamma H=-J_{2}+\gamma H$, so is not independent.

Remark 7.2 (transformation of potentials). Notice that the change of coordinates (7.2) transforms the Hamiltonian of (7.1) to that of (7.3), preserving the form of the 2-dimensional metric $D_{2}$, but changing the potential.

Remark 7.3 (reduction with $\beta=0$ ). To get this reduction, we can apply the involution $\iota_{23}$ to the case $\alpha=0$.

\subsection{Reductions when $\gamma=0$}

Now we have

$$
\varphi=\frac{q_{2}^{2} q_{3}^{2}}{\alpha q_{2}^{2}+\beta q_{3}^{2}} \quad \text { and } \quad \psi=-2 \beta \frac{q_{1}^{2}+q_{3}^{2}}{q_{2}^{2}}
$$

so still have the involutive symmetry $\iota_{23}$ (extended to include $\alpha \leftrightarrow \beta$ ). We have also gained additional Killing vectors, giving us the isometry algebra $\mathfrak{g}_{1}=\left\langle e_{1}, h_{1}, f_{1}\right\rangle$. We can therefore reduce with respect to either $h_{1}$ or $f_{1}$. 


\subsubsection{Reduction using the isometry $K_{2}=h_{1}$}

We now want to reduce our Hamiltonian (4.1) to the submanifold $h_{1}=\mu$. To do this, we choose canonical coordinates $Q_{i}, P_{i}$ so that $h_{1}=P_{3}$, which makes $H$ independent of $Q_{3}$. It is easy to see that

$$
\left\{\frac{q_{1}}{q_{3}}, h_{1}\right\}=0, \quad\left\{\frac{q_{2}}{q_{3}}, h_{1}\right\}=0, \quad\left\{-\frac{1}{2} \log q_{3}, h_{1}\right\}=1,
$$

so clearly we should choose $Q_{3}=-\frac{1}{2} \log q_{3}$ and then some convenient functions of $z_{1}=\frac{q_{1}}{q_{3}}$, $z_{2}=\frac{q_{2}}{q_{3}}$ for $Q_{1}, Q_{2}$. To obtain a diagonal metric on our reduced space we need the orthogonality relation $\sum_{i, j=1}^{3} g^{i j} \partial_{i} Q_{1} \partial_{j} Q_{2}=0$. We choose $Q_{1}=\frac{q_{1}}{q_{3}}$ and $Q_{2}=s\left(\frac{q_{1}}{q_{3}}, \frac{q_{2}}{q_{3}}\right)$, leading to

$$
\left(\left(z_{1}^{2}+1\right) \partial_{z_{1}}+z_{1} z_{2} \partial_{z_{2}}\right) s\left(z_{1}, z_{2}\right)=0 \quad \Rightarrow \quad s\left(z_{1}, z_{2}\right)=f\left(\frac{z_{2}}{\sqrt{z_{1}^{2}+1}}\right) .
$$

With the point transformation

$$
Q_{1}=\frac{q_{1}}{q_{3}}, \quad Q_{2}=\frac{\sqrt{q_{1}^{2}+q_{3}^{2}}}{q_{2}}, \quad Q_{3}=-\frac{1}{2} \log q_{3},
$$

we find

$$
H=\sum_{i, j=1}^{3} \hat{g}^{i j} P_{i} P_{j}, \quad \hat{g}^{i j}=\frac{1}{\delta}\left(\begin{array}{ccc}
\left(1+Q_{1}^{2}\right)^{2} & 0 & \frac{1}{2} Q_{1}\left(1+Q_{1}^{2}\right) \\
0 & Q_{2}^{2}\left(1+Q_{2}^{2}\right) & -\frac{1}{2} Q_{2} \\
\frac{1}{2} Q_{1}\left(1+Q_{1}^{2}\right) & -\frac{1}{2} Q_{2} & \frac{1}{4}\left(1+Q_{1}^{2}\right)
\end{array}\right),
$$

where $\delta=\alpha\left(1+Q_{1}^{2}\right)+\beta Q_{2}^{2}$.

\section{The resulting $2 \mathrm{D}$ system}

By setting $P_{3}=\mu$, we can consider our Hamiltonian as 2-dimensional, with (upper index) metric $\hat{g}^{i j}$, given by the $2 \times 2$ diagonal matrix in $(7.4 \mathrm{~b})$. However, since we wish to consider this as an embedding into 3 dimensions, it is better to leave $P_{3}$ in the formulae, remembering that we can always set $P_{3}=\mu$ if we wish to consider the 2-dimensional reduction. The quadratic terms $P_{i} P_{3}$ are incorporated in the formulae by "completing the square", writing the Hamiltonian as one with electro-magnetic, vector potential:

$$
H=\sum_{i=1}^{2} \hat{g}^{i i}\left(P_{i}-A_{i} P_{3}\right)^{2}+h\left(Q_{1}, Q_{2}\right) P_{3}^{2},
$$

where

$$
A_{1}=\frac{-Q_{1}}{2\left(1+Q_{1}^{2}\right)}, \quad A_{2}=\frac{1}{2 Q_{2}\left(1+Q_{2}^{2}\right)}, \quad h=\frac{Q_{2}^{2}}{4\left(1+Q_{1}^{2}\right)\left(\alpha\left(1+Q_{1}^{2}\right)+\beta Q_{2}^{2}\right)} .
$$

Since, for these particular $A_{i}$ we have $\left\{P_{1}-A_{1} P_{3}, P_{2}-A_{2} P_{3}\right\}=\left(\partial_{1} A_{2}-\partial_{2} A_{1}\right) P_{3}=0$, we may redefine the momenta (a gauge transformation) by $\hat{P}_{i}=P_{i}-A_{i} P_{3}$, so that (7.5) takes the form $H=\sum_{i=1}^{2} \hat{g}^{i i} \hat{P}_{i}^{2}+\hat{h}\left(Q_{1}, Q_{2}\right) P_{3}^{2}$. To incorporate this into the canonical transformation, we must adjust $Q_{3}$ :

$$
\hat{Q}_{3}=Q_{3}+M_{1}\left(Q_{1}\right)+M_{2}\left(Q_{2}\right) \quad \text { so that } \quad\left\{\hat{Q}_{3}, P_{i}-A_{i}\left(Q_{i}\right) P_{3}\right\}=0,
$$


leading to $M_{1}=-\frac{1}{4} \log \left(1+Q_{1}^{2}\right), M_{2}=\frac{1}{4} \log \left(\frac{Q_{2}^{2}}{1+Q_{2}^{2}}\right)$, giving the point transformation (dropping the hats):

$$
Q_{1}=\frac{q_{1}}{q_{3}}, \quad Q_{2}=\frac{\sqrt{q_{1}^{2}+q_{3}^{2}}}{q_{2}}, \quad Q_{3}=-\frac{1}{4} \log \left(q_{1}^{2}+q_{2}^{2}+q_{3}^{2}\right) .
$$

In the original 3 -dimensional setting we had the isometry algebra $\mathfrak{g}_{1}=\left\langle e_{1}, h_{1}, f_{1}\right\rangle$. Since neither of $e_{1}, f_{1}$ commute with $h_{1}$, we cannot reduce these to the 2 -dimensional setting. However, since $\left\{e_{1} f_{1}, h_{1}\right\}=0$, we can reduce this quadratic element, which takes the form

$$
e_{1} f_{1}=T_{1}^{2}+\left(P_{1}+\frac{Q_{1} Q_{2} P_{2}}{1+Q_{1}^{2}}\right) Q_{1} P_{3}, \quad T_{1}=\frac{1}{Q_{2}} \sqrt{\frac{1+Q_{2}^{2}}{1+Q_{1}^{2}}}\left(\left(1+Q_{1}^{2}\right) P_{1}+Q_{1} Q_{2} P_{2}\right)
$$

where $T_{1}$ is a first order, first integral of the kinetic energy $H_{0}=\sum_{i=1}^{2} \hat{g}^{i i} P_{i}^{2}$.

\section{Adapting coordinates to $T_{1}$}

By noting that

$$
y_{1}=\frac{Q_{2}}{\sqrt{1+Q_{1}^{2}}}, \quad y_{2}=\log \left(\frac{Q_{1} Q_{2}}{\sqrt{1+Q_{1}^{2}}}+\sqrt{1+Q_{2}^{2}}\right)
$$

satisfy $\left\{y_{1}, T_{1}\right\}=0,\left\{y_{2}, T_{1}\right\}=1$, we may choose $\left(y_{1}, y_{2}+B\left(y_{1}\right)\right)$ as new coordinates, with $B\left(y_{1}\right)$ chosen so that the resulting metric is diagonal. In this way we find

$$
u=\frac{Q_{2}}{\sqrt{1+Q_{1}^{2}}}, \quad v=\log \left(\frac{Q_{1} Q_{2}+\sqrt{\left(1+Q_{1}^{2}\right)\left(1+Q_{2}^{2}\right)}}{\sqrt{1+Q_{1}^{2}+Q_{2}^{2}}}\right),
$$

in which coordinates $T_{1}$ and $H$ take the form

$$
T_{1}=p_{v}, \quad H=\frac{1}{\alpha+\beta u^{2}}\left(u^{2}\left(1+u^{2}\right) p_{u}^{2}+\frac{u^{2}}{1+u^{2}} p_{v}^{2}+\frac{u^{2} \operatorname{sech}^{2} v p_{w}^{2}}{4\left(1+u^{2}\right)}\right) .
$$

The final step is to put $H_{0}$ into conformal form $H_{0}=\bar{\varphi}(\bar{u})\left(p_{\bar{u}}^{2}+p_{\bar{v}}^{2}\right)$, in which case, the LaplaceBeltrami operator takes a very simple form: $L_{b}=\varphi(\bar{u}, \bar{v})\left(\partial_{\bar{u}}^{2} \pm \partial_{\bar{v}}^{2}\right)$, with no first order terms. As a consequence, the coordinates $\bar{u}$ and $\bar{v}$ satisfy $L_{b} \bar{u}=L_{b} \bar{v}=0$. Solutions of these equations are

$$
\bar{u}=\arctan (u), \quad \bar{v}=v,
$$

which, dropping bars, gives

$$
T_{1}=p_{v}, \quad H=\frac{\sin ^{2}(2 u)}{2(\alpha+\beta)+2(\alpha-\beta) \cos (2 u)}\left(p_{u}^{2}+p_{v}^{2}+\frac{1}{4} \operatorname{sech}^{2} v p_{w}^{2}\right),
$$

which has the $D_{4}$ kinetic energy, with potential (equivalent to the " $b_{3}$ " part of Case B in [9]).

We can see that $h_{1}$ commutes with the 6 functions

$$
e_{1} f_{1}, e_{2} f_{2}, e_{3} f_{3}, h_{2}, h_{3} \text {, and } h_{4},
$$

which correspond to the 6-dimensional conformal algebra of our $2 \mathrm{D}$ kinetic energy $H_{0}$. In the present coordinates, these take the form

$$
\begin{aligned}
& T_{1}=p_{v}, \quad T_{2}=p_{u} \cosh v \sin u+p_{v} \sinh v \cos u, \quad T_{3}=p_{u} \cosh v \cos u-p_{v} \sinh v \sin u, \\
& T_{4}=p_{u} \sinh v \sin u+p_{v} \cosh v \cos u, \quad T_{5}=p_{u} \sinh v \cos u-p_{v} \cosh v \sin u, \quad T_{6}=p_{u} .
\end{aligned}
$$


We have seen that both $J_{1}=F_{1}$ and $J_{2}=e_{1} f_{1}$ commute with $h_{1}$ (in the $3 \mathrm{D}$ space), so can be reduced to the 2 -dimensional setting. We therefore have that

$$
\begin{aligned}
& J_{1}=2 T_{2}^{2}+\beta(\cos 2 u-\cosh 2 v) \operatorname{sech}^{2} u H-\frac{1}{4} \cos ^{2} u \operatorname{sech}^{2} v p_{w}^{2}, \\
& J_{2}=T_{1}^{2}-\frac{1}{4} \tanh ^{2} v p_{w}^{2},
\end{aligned}
$$

commute with $H$.

\subsubsection{Reduction using the isometry $K_{3}=f_{1}$}

We now want to reduce our Hamiltonian (4.1) to the submanifold $f_{1}=\mu$, so choose canonical coordinates $Q_{i}, P_{i}$ so that $f_{1}=P_{3}$, which makes $H$ independent of $Q_{3}$. It is easy to see that we should choose $Q_{3}=\frac{q_{1}}{q_{1}^{2}+q_{2}^{2}+q_{3}^{2}}$ and then some convenient functions of $z_{1}=\frac{q_{3}}{q_{2}}$ and $z_{2}=\frac{q_{1}^{2}+q_{2}^{2}+q_{3}^{2}}{q_{2}}$ for $Q_{1}$ and $Q_{2}$. To obtain a diagonal metric on our reduced space we need the orthogonality relation $\sum_{i, j=1}^{3} g^{i j} \partial_{i} Q_{1} \partial_{j} Q_{2}=0$, which leads us to

$$
Q_{1}=\frac{q_{3}}{q_{2}}, \quad Q_{2}=\frac{q_{1}^{2}+q_{2}^{2}+q_{3}^{2}}{\sqrt{q_{2}^{2}+q_{3}^{2}}}, \quad Q_{3}=\frac{q_{1}}{q_{1}^{2}+q_{2}^{2}+q_{3}^{2}},
$$

and

$$
H=\frac{Q_{1}^{2}}{\alpha+\beta Q_{1}^{2}}\left(\left(Q_{1}^{2}+1\right) P_{1}^{2}+\frac{Q_{2}^{2}}{Q_{1}^{2}+1} P_{2}^{2}+\frac{P_{3}^{2}}{Q_{2}^{2}\left(Q_{1}^{2}+1\right)}\right) .
$$

From the Casimir $\mathcal{C}=e_{1} f_{1}+\frac{1}{4} h_{1}^{2}$, of our isometry algebra, we obtain

$$
\mathcal{C}=T_{1}^{2}+\frac{P_{3}^{2}}{Q_{2}^{2}}
$$

where $T_{1}=Q_{2} P_{2}$ corresponds to a Killing vector on the reduced space.

\section{Adapting coordinates to $T_{1}$}

We have

$$
\left\{Q_{1}, T_{1}\right\}=\left\{Q_{3}, T_{1}\right\}=0 \quad \text { and } \quad\left\{\log \left(Q_{2}\right), T_{1}\right\}=1,
$$

so must build coordinates out of these, such that the kinetic energy $H_{0}$ is transformed into conformal form $H_{0}=\varphi(u)\left(p_{u}^{2}+p_{v}^{2}\right)$. This is achieved by the choice

$$
u=\arctan \left(Q_{1}\right), \quad v=\log \left(Q_{2}\right), \quad w=Q_{3} \quad \Rightarrow \quad T_{1}=p_{v}
$$

and

$$
H=\frac{\sin ^{2}(2 u)}{2(\alpha+\beta)+2(\alpha-\beta) \cos (2 u)}\left(p_{u}^{2}+p_{v}^{2}+\mathrm{e}^{-2 v} p_{w}^{2}\right)
$$

which has the $D_{4}$ kinetic energy, with potential (equivalent to the " $a_{1}$ " part of Case A in [9]). 


\section{The 6-dimensional conformal algebra}

We can identify 3 linear and 3 quadratic conformal elements in the 3D space, which commute with $f_{1}$, so can be reduced to the 2 -dimensional setting:

$$
e_{1} f_{1}+\frac{1}{4} h_{1}^{2}, f_{2}, f_{3}, h_{4}, e_{2} f_{2}-\frac{1}{2} h_{2}^{2}, e_{3} f_{3}+\frac{1}{2} h_{3}^{2},
$$

leading to

$$
\begin{aligned}
& T_{1}=p_{v}, \quad T_{2}=\mathrm{e}^{v}\left(\sin u p_{u}+\cos u p_{v}\right), \quad T_{3}=\mathrm{e}^{v}\left(\cos u p_{u}-\sin u p_{v}\right), \\
& T_{4}=p_{u}, \quad T_{5}=\mathrm{e}^{-v}\left(\sin u p_{u}-\cos u p_{v}\right), \quad T_{6}=\mathrm{e}^{-v}\left(\cos u p_{u}+\sin u p_{v}\right),
\end{aligned}
$$

with $T_{1}$ being the sole isometry.

The Hamiltonian (7.8) has 2 independent quadratic integrals, derived from the Casimir (7.7) and the former integral $F_{5}$ (see (4.6)), which can be written in terms of the above conformal elements:

$$
J_{1}=T_{1}^{2}+\mathrm{e}^{-2 v} p_{w}^{2}, \quad J_{2}=4 T_{2}^{2}-4 \beta \mathrm{e}^{2 v} \sec ^{2} u H .
$$

\subsubsection{The relationship between these reductions}

If we consider the $h_{1}$ reduction to have coordinates $(\bar{u}, \bar{v}, \bar{w})$, then combining the transformations, we obtain

$$
u=\bar{u}, \quad v=2 \bar{w}+\log (\cosh \bar{v}), \quad w=\mathrm{e}^{2 \bar{w}} \tanh \bar{v},
$$

and the Hamiltonian (7.8) is transformed onto that of (7.6) (with bars). This transformation preserves the form of the 2-dimensional kinetic energy, but changes the potential, thus relating two of the potentials obtained in [9].

\section{Reductions of the system of Section 4.2}

We saw in Section 4.2 that the Hamiltonian

$$
H=\frac{1}{\alpha q_{2}+\beta q_{3}+\gamma}\left(p_{1}^{2}+p_{2}^{2}+p_{3}^{2}\right)
$$

admitted the 3 -dimensional isometry algebra (4.8a), with Poisson relations (4.8b). In this section we reduce this Hamiltonian to a $2 \mathrm{D}$ space in two different ways, using the isometries $K_{2}$ and $K_{3}$ respectively.

\subsection{Reduction using the isometry $K_{2}=\beta e_{2}-\alpha e_{3}$}

We see that

$$
\left\{\alpha q_{2}+\beta q_{3}, K_{2}\right\}=\left\{q_{1}, K_{2}\right\}=0 \quad \text { and } \quad\left\{\frac{q_{2}}{\beta}, K_{2}\right\}=1,
$$

and we need to choose new coordinates $\left(Q_{1}, Q_{2}, Q_{3}\right)$, such that $K_{2}=P_{3}$ (so the new $H$ is independent of $Q_{3}$ ) and $H$ takes the form

$$
H=\tilde{\varphi}\left(Q_{1}, Q_{2}\right)\left(P_{1}^{2}+P_{2}^{2}\right)+\tilde{V}\left(Q_{1}, Q_{2}\right) P_{3}^{2},
$$


which can be achieved in stages as described in Section 7 or by the single canonical transformation generated by

$$
S=\frac{\left(\alpha q_{2}+\beta q_{3}+\gamma\right)}{\left(\alpha^{2}+\beta^{2}\right)^{\frac{1}{3}}} P_{1}+\left(\alpha^{2}+\beta^{2}\right)^{\frac{1}{6}} q_{1} P_{2}+\left(\frac{q_{2}}{\beta}-\frac{\alpha\left(\alpha q_{2}+\beta q_{3}+\gamma\right)}{\beta\left(\alpha^{2}+\beta^{2}\right)}\right) P_{3},
$$

which transforms the Hamiltonian to

$$
H=\frac{1}{Q_{1}}\left(P_{1}^{2}+P_{2}^{2}\right)+\frac{P_{3}^{2}}{\left(\alpha^{2}+\beta^{2}\right)^{\frac{4}{3}} Q_{1}},
$$

so the entire Hamiltonian (not just the kinetic energy) has inherited the symmetry $K_{1}=\left(\alpha^{2}+\right.$ $\left.\beta^{2}\right)^{\frac{1}{6}} P_{2}$, which is a result of the property $\left\{K_{1}, K_{2}\right\}=0$.

Since $P_{3}$ is a first integral, we can reduce to the 2 -dimensional space with $P_{3}=\mu$. Again, we can derive the 6 -dimensional conformal algebra from our original 10-dimensional algebra (2.2), by considering linear and quadratic conformal elements which commute with $K_{2}$. The 3 linear elements

$$
\alpha e_{2}+\beta e_{3}, e_{1}, \alpha h_{2}+\beta h_{3} \quad \text { give us } \quad T_{1}=P_{1}, \quad T_{2}=P_{2}, \quad T_{3}=Q_{2} P_{1}-Q_{1} P_{2},
$$

while the 3 quadratic elements

$$
\alpha K_{1} h_{1}+h_{3} K_{2}, \quad 8\left(\alpha e_{2}+\beta e_{3}\right)\left(\alpha f_{2}+\beta f_{3}\right)-\left(\alpha^{2}+\beta^{2}\right) h_{4}^{2}, \quad 4\left(\alpha^{2}+\beta^{2}\right) e_{1} f_{1}-K_{3}^{2},
$$

factorise (when $P_{3}=0$ ) to give the 3 remaining conformal elements:

$$
\begin{aligned}
& T_{4}=Q_{1} P_{1}+Q_{2} P_{2}, \quad T_{5}=\left(Q_{1}^{2}-Q_{2}^{2}\right) P_{1}+2 Q_{1} Q_{2} P_{2}, \\
& T_{6}=2 Q_{1} Q_{2} P_{1}+\left(Q_{2}^{2}-Q_{1}^{2}\right) P_{2} .
\end{aligned}
$$

The 2-dimensional Hamiltonian (8.2) (with $P_{3}=\mu$ ) is the $D_{1}$ kinetic energy, with potential (equivalent to the " $b_{2}$ " part of Case 1 in $[10]$ ).

\subsubsection{The quadratic integrals}

We consider the 2 functions (4.7a) and see that $\left\{F_{2}, K_{2}\right\}=0$, but $\left\{F_{1}, K_{2}\right\} \neq 0$. However, it is easy to see that $\left\{\left(\alpha^{2}+\beta^{2}\right) F_{1}-\beta K_{1} K_{3}, K_{2}\right\}=\left\{K_{1}, K_{2}\right\}=0$. Noting that

$$
\left(\alpha^{2}+\beta^{2}\right) F_{1}-\beta K_{1} K_{3}=\alpha K_{1}\left(\alpha h_{2}+\beta h_{3}\right)-\frac{1}{2} \alpha\left(\alpha^{2}+\beta^{2}\right) q_{1}^{2} H
$$

and that $\alpha h_{2}+\beta h_{3} \mapsto 2 \gamma\left(\alpha^{2}+\beta^{2}\right)^{\frac{1}{6}} T_{2}+2\left(\alpha^{2}+\beta^{2}\right)^{\frac{1}{2}} T_{3}$ (under the action of the transformation generated by (8.1)) this integral reduces to

$$
J_{1}=T_{2} T_{3}-\frac{1}{4} Q_{2}^{2} H=P_{2}\left(Q_{2} P_{1}-Q_{1} P_{2}\right)-\frac{1}{4} Q_{2}^{2} H .
$$

The function $F_{2}$ is more straightforward and reduces to

$$
J_{2}=T_{1} T_{2}-\frac{Q_{2}}{2} H=P_{1} P_{2}-\frac{Q_{2}}{2} H .
$$

In both these formulae, $H$ is the full (8.2) (with potential) and $\left\{J_{i}, H\right\}=0$, for each $i$, with $\left\{J_{1}, P_{2}\right\}=4 J_{2}$. 


\subsection{Reduction using the isometry $K_{3}=\beta h_{2}-\alpha h_{3}$}

We see that

$$
\begin{aligned}
& \left\{\alpha q_{2}+\beta q_{3}, K_{3}\right\}=\left\{q_{1}^{2}+q_{2}^{2}+q_{3}^{2}, K_{3}\right\}=0 \quad \text { and } \\
& \left\{\frac{1}{2 \sqrt{\alpha^{2}+\beta^{2}}} \tan ^{-1}\left(\frac{\beta q_{2}-\alpha q_{3}}{q_{1} \sqrt{\alpha^{2}+\beta^{2}}}\right), K_{3}\right\}=1,
\end{aligned}
$$

and we again need to choose new coordinates $\left(Q_{1}, Q_{2}, Q_{3}\right)$, such that $K_{2}=P_{3}$ (so the new $H$ is independent of $Q_{3}$ ) and $H$ takes the form

$$
H=\tilde{\varphi}\left(Q_{1}, Q_{2}\right)\left(P_{1}^{2}+P_{2}^{2}\right)+\tilde{V}\left(Q_{1}, Q_{2}\right) P_{3}^{2},
$$

which can be achieved in stages as described in Section 7 or by the single canonical transformation generated by

$$
\begin{aligned}
S= & \frac{\alpha q_{2}+\beta q_{3}+\gamma}{\left(\alpha^{2}+\beta^{2}\right)^{\frac{1}{3}}} P_{1}+\frac{\left(\alpha^{2}+\beta^{2}\right) q_{1}^{2}+\left(\beta q_{2}-\alpha q_{3}\right)^{2}}{\left(\alpha^{2}+\beta^{2}\right)^{\frac{1}{3}}} P_{2} \\
& +\frac{1}{2 \sqrt{\alpha^{2}+\beta^{2}}} \tan ^{-1}\left(\frac{\beta q_{2}-\alpha q_{3}}{q_{1} \sqrt{\alpha^{2}+\beta^{2}}}\right) P_{3},
\end{aligned}
$$

which transforms the Hamiltonian to

$$
H=\frac{1}{Q_{1}}\left(P_{1}^{2}+P_{2}^{2}\right)+\frac{P_{3}^{2}}{4\left(\alpha^{2}+\beta^{2}\right) Q_{1} Q_{2}^{2}},
$$

so this Hamiltonian has not inherited the symmetry $K_{1}$, but the kinetic energy has acquired the symmetry $P_{2}$, which is the reduction of $K_{1}$ to the $2 \mathrm{D}$ space, corresponding to the level set $P_{3}=\mu$.

Again, we can derive the 6-dimensional conformal algebra from our original 10-dimensional algebra (2.2), by considering linear and quadratic conformal elements which commute with $K_{3}$. The 3 linear elements

$$
\begin{aligned}
& \alpha e_{2}+\beta e_{3}, h_{1}, \alpha f_{2}+\beta f_{3} \quad \text { give us } \\
& T_{1}=P_{1}, \quad T_{4}=Q_{1} P_{1}+Q_{2} P_{2}, \quad T_{5}=\left(Q_{1}^{2}-Q_{2}^{2}\right) P_{1}+2 Q_{1} Q_{2} P_{2},
\end{aligned}
$$

while the 3 quadratic elements

$$
4\left(h_{2}^{2}+h_{3}^{2}\right)+h_{4}^{2}, \quad K_{2}^{2}+\left(\alpha^{2}+\beta^{2}\right) K_{1}^{2}, \quad 4\left(\alpha^{2}+\beta^{2}\right) f_{1}^{2}\left(\beta f_{2}-\alpha f_{3}\right)^{2},
$$

simplify (when $P_{3}=0$ ) to give the 3 remaining conformal elements:

$$
T_{2}=P_{2}, \quad T_{3}=Q_{2} P_{1}-Q_{1} P_{2}, \quad T_{6}=2 Q_{1} Q_{2} P_{1}+\left(Q_{2}^{2}-Q_{1}^{2}\right) P_{2} .
$$

The 2-dimensional Hamiltonian (8.3) (with $P_{3}=\mu$ ) is the $D_{1}$ kinetic energy, with potential (equivalent to the " $b_{3}$ " part of Case 1 in $[10]$ ).

\subsection{The relationship between these reductions}

For this section we change notation for the reduction of Section 8.1, writing the 3D coordinates as $(u, v, w)$ :

$$
\begin{aligned}
& H_{u}=\frac{1}{u}\left(p_{u}^{2}+p_{v}^{2}\right)+\frac{p_{w}^{2}}{\left(\alpha^{2}+\beta^{2}\right)^{\frac{4}{3}} u}, \quad J_{1 u}=p_{v}\left(v p_{u}-u p_{v}\right)-\frac{1}{4} v^{2} H_{u}, \\
& J_{2 u}=p_{u} p_{v}-\frac{v}{2} H_{u} .
\end{aligned}
$$


Composing the two point transformations $(u, v, w) \mapsto\left(q_{1}, q_{2}, q_{3}\right) \mapsto\left(Q_{1}, Q_{2}, Q_{3}\right)$, we obtain

$$
\begin{aligned}
& u=Q_{1}, \quad v=Q_{2} \cos \left(2 \sqrt{\alpha^{2}+\beta^{2}} Q_{3}\right), \\
& w=\frac{1}{\left(\alpha^{2}+\beta^{2}\right)^{\frac{2}{3}}} Q_{2} \sin \left(2 \sqrt{\alpha^{2}+\beta^{2}} Q_{3}\right)-\frac{\alpha}{\beta\left(\alpha^{2}+\beta^{2}\right)} .
\end{aligned}
$$

Under the corresponding canonical transformation of the full 6-dimensional phase space, the Hamiltonian $H_{u}$ is transformed to $H_{Q}$, given by (8.3). This means that it preserves the form of the kinetic energy (associated with $D_{1}$ ) and changes the " $b_{2}$ " part of the potential of Case 1 in $[10]$ to the " $b_{3}$ " part.

Any other integral $F_{u}$ is transformed to

$$
F_{Q}=F_{Q}^{(0)}+F_{Q}^{(c)} \cos \left(k Q_{3}\right)+F_{Q}^{(s)} \sin \left(k Q_{3}\right), \quad \text { for some } \quad k .
$$

In the full 6-dimensional phase space, $\left\{H_{Q}, F_{Q}\right\}=0$ (noting that the $P_{3}^{2}$ term in $H_{Q}$ will interact with $Q_{3}$ ), but in the 2-dimensional reduction (with $P_{3}=\mu$ ), we have $\left\{H_{Q}, F_{Q}^{(0)}\right\}=0$.

Carrying this out for $J_{1 u}, J_{2 u}$, we find

$$
J_{1 Q}^{(0)}=P_{2}\left(Q_{2} P_{1}-Q_{1} P_{2}\right)-\frac{Q_{1} P_{3}^{2}}{4\left(\alpha^{2}+\beta^{2}\right) Q_{2}^{2}}-\frac{1}{4} Q_{2}^{2} H_{Q}, \quad J_{2 Q}^{(0)}=0 .
$$

The function $J_{2 u}^{2}$ would lead to something nontrivial, but it would be quartic. However, we can use the Casimir (4.8c) of the original isometry algebra:

$$
\mathcal{C}_{K}=\left(\alpha^{2}+\beta^{2}\right)^{\frac{4}{3}} p_{v}^{2}+p_{w}^{2}=\left(\alpha^{2}+\beta^{2}\right)^{\frac{4}{3}}\left(P_{2}^{2}+\frac{P_{3}^{2}}{4\left(\alpha^{2}+\beta^{2}\right) Q_{2}^{2}}\right) .
$$

We have thus found that the system (8.4) (together with combinations of its linear integrals) can be transformed onto the system

$$
\begin{aligned}
& H_{Q}=\frac{1}{Q_{1}}\left(P_{1}^{2}+P_{2}^{2}\right)+\frac{P_{3}^{2}}{4\left(\alpha^{2}+\beta^{2}\right) Q_{1} Q_{2}^{2}}, \\
& R_{1 Q}=P_{2}\left(Q_{2} P_{1}-Q_{1} P_{2}\right)-\frac{Q_{1} P_{3}^{2}}{4\left(\alpha^{2}+\beta^{2}\right) Q_{2}^{2}}-\frac{1}{4} Q_{2}^{2} H_{Q}, \\
& R_{2 Q}=P_{2}^{2}+\frac{P_{3}^{2}}{4\left(\alpha^{2}+\beta^{2}\right) Q_{2}^{2}},
\end{aligned}
$$

corresponding to two particular parametric reductions of the potential of Case 1 in [10].

\section{Reductions of the system of Section 5.1}

We now consider reductions of the Hamiltonian

$$
H=\frac{q_{2}^{2} q_{3}^{2} \sqrt{q_{1}^{2}+q_{2}^{2}}}{\left(\alpha q_{2}^{2}+\beta q_{3}^{2}\right) \sqrt{q_{1}^{2}+q_{2}^{2}}+\gamma q_{1} q_{3}^{2}}\left(p_{1}^{2}+p_{2}^{2}+p_{3}^{2}\right) .
$$

We found that we had three cases, corresponding to $\alpha=0$ or $\beta=0$ or $\gamma=0$. However, $\gamma=0$ is just the Hamiltonian discussed in Sections 4.1.2 and 7.2, so we are left with just the first two. Our first example is the case with $\alpha=0$, which has a 3-dimensional isometry algebra and reduces to a Hamiltonian with Darboux-Koenigs metric $D_{4}$. However, in Section 9.2, we consider the case $\beta=0$, which has only one linear integral $h_{1}$, with the consequence that the reduced metric has no Killing vectors at all. Nevertheless, the reduced Hamiltonian commutes with a pair of functions, one quadratic and the other quartic, so is a maximally super-integrable system which is outside the Darboux-Koenigs classification. 


\subsection{The case when $\alpha=0$}

We now consider the Hamiltonian

$$
H=\frac{q_{2}^{2} \sqrt{q_{1}^{2}+q_{2}^{2}}}{\beta \sqrt{q_{1}^{2}+q_{2}^{2}}+\gamma q_{1}}\left(p_{1}^{2}+p_{2}^{2}+p_{3}^{2}\right)
$$

which has the isometry algebra $K_{1}=h_{1}, K_{2}=e_{3}, K_{3}=f_{3}$, satisfying

$$
\left\{K_{1}, K_{2}\right\}=-2 K_{2}, \quad\left\{K_{1}, K_{3}\right\}=2 K_{3}, \quad\left\{K_{2}, K_{3}\right\}=-2 K_{1} .
$$

We can therefore reduce with respect to either $K_{1}$ or $K_{3}$. Reduction with respect to $K_{2}$ would be equivalent to that with respect to $K_{3}$ through the action of the involution $\iota_{e f}$.

\subsubsection{Reduction using the isometry $K_{1}$}

Since $K_{1}=h_{1}$, we can again use the transformation (7.4a):

$$
Q_{1}=\frac{q_{1}}{q_{3}}, \quad Q_{2}=\frac{\sqrt{q_{1}^{2}+q_{3}^{2}}}{q_{2}}, \quad Q_{3}=-\frac{1}{2} \log q_{3},
$$

this time giving

$$
\begin{aligned}
H= & \frac{Q_{1}^{2}}{\beta\left(1+Q_{1}^{2}\right)+\gamma \sqrt{1+Q_{1}^{2}}}\left(\left(1+Q_{1}^{2}\right)^{2}\left(P_{1}+\frac{Q_{1} P_{3}}{2\left(1+Q_{1}^{2}\right)}\right)^{2}\right. \\
& \left.+\left(1+Q_{2}^{2}\right)\left(P_{2}+\frac{Q_{2} P_{3}}{2\left(1+Q_{2}^{2}\right)}\right)^{2}+\frac{P_{3}^{2}}{4\left(1+Q_{2}^{2}\right)}\right)
\end{aligned}
$$

Here we have already "completed the square" to give the vector potential

$$
\left(A_{1}, A_{2}\right)=\left(-\frac{Q_{1}}{2\left(1+Q_{1}^{2}\right)},-\frac{Q_{2}}{2\left(1+Q_{2}^{2}\right)}\right),
$$

satisfying

$$
\left\{P_{1}-A_{1} P_{3}, P_{2}-A_{2} P_{3}\right\}=\left(\partial_{1} A_{2}-\partial_{2} A_{1}\right) P_{3}=0
$$

so we can use these to define new momenta $\left(\tilde{P}_{1}, \tilde{P}_{2}\right)$, but must adjust the definition of $Q_{3}$ in order to keep the transformation canonical:

$$
\left\{Q_{3}+M_{1}\left(Q_{1}\right)+M_{2}\left(Q_{2}\right), P_{i}-A_{i} P_{3}\right\}=0 \quad \Rightarrow \quad M_{1}^{\prime}\left(Q_{1}\right)=A_{1}, \quad M_{2}^{\prime}\left(Q_{2}\right)=A_{2},
$$

leading to the point transformation

$$
Q_{1}=\frac{q_{1}}{q_{3}}, \quad Q_{2}=\frac{\sqrt{q_{1}^{2}+q_{3}^{2}}}{q_{2}}, \quad Q_{3}=-\frac{1}{4} \log \left(q_{1}^{2}+q_{2}^{2}+q_{3}^{2}\right),
$$

now giving

$$
H=\frac{Q_{1}^{2}}{\beta\left(1+Q_{1}^{2}\right)+\gamma \sqrt{1+Q_{1}^{2}}}\left(\left(1+Q_{1}^{2}\right)^{2} P_{1}^{2}+\left(1+Q_{2}^{2}\right) P_{2}^{2}+\frac{P_{3}^{2}}{4\left(1+Q_{2}^{2}\right)}\right) .
$$


Since $\left\{K_{2} K_{3}, K_{1}\right\}=0$, this quantity defines a Killing vector for the 2-dimensional metric, associated with this Hamiltonian:

$$
K_{2} K_{3}=2\left(T_{1}^{2}-\frac{Q_{2}^{2} P_{3}^{2}}{4\left(1+Q_{2}^{2}\right)}\right), \quad \text { with } \quad T_{1}=\sqrt{\left(1+Q_{2}^{2}\right)} P_{2},
$$

where $T_{1}$ is a first order, first integral of the $2 \mathrm{D}$ kinetic energy $H_{0}$.

We choose coordinates $(u, v, w)$ so that $T_{1}=p_{v}$ and $H_{0}=\tilde{\varphi}(u)\left(p_{u}^{2}+p_{v}^{2}\right)$ :

$$
Q_{1}=\tan u, \quad Q_{2}=\sinh v, \quad Q_{3}=w,
$$

giving

$$
H=\frac{\sin ^{2} u}{\beta+\gamma \cos u}\left(p_{u}^{2}+p_{v}^{2}+\frac{1}{4} \operatorname{sech}^{2} v p_{w}^{2}\right)
$$

which has the $D_{4}$ kinetic energy, with potential (equivalent to the " $b_{3}$ " part of Case B in [9]).

\section{The conformal algebra and quadratic integrals}

We can see that $K_{1}=h_{1}$ commutes with the 6 functions

$$
e_{3} f_{3}, \quad e_{1} f_{1}, \quad e_{2} f_{2}, \quad h_{2}, \quad h_{3}, \quad \text { and } h_{4},
$$

which correspond to the 6-dimensional conformal algebra of our $2 \mathrm{D}$ kinetic energy $H_{0}$. In the present coordinates, these take the form

$$
\begin{array}{lll}
T_{1}=p_{v}, & T_{2}=p_{u} \cosh v \sin u+p_{v} \sinh v \cos u, & T_{3}=p_{u} \cosh v \cos u-p_{v} \sinh v \sin u, \\
T_{4}=p_{u}, & T_{5}=p_{u} \sinh v \sin u+p_{v} \cosh v \cos u, & T_{6}=p_{u} \sinh v \cos u-p_{v} \cosh v \sin u
\end{array}
$$

The element $T_{1}$ is an invariant of the kinetic energy, but the full expression for $K_{2} K_{3}=e_{3} f_{3}$ is a quadratic integral for $H$ :

$$
J_{1}=\frac{1}{2} e_{3} f_{3}=T_{1}^{2}-\frac{1}{4} \tanh ^{2} v p_{w}^{2} .
$$

We also have that $\left\{F_{3}, K_{1}\right\}=\left\{F_{4}, K_{1}\right\}=0$ (see Table 8), but $F_{3}=4 J_{1}-4 \beta H+p_{w}^{2}$, so is not independent. However $F_{4}$ is independent and takes the form

$$
J_{2}=F_{4}=-8 T_{4} T_{6}+2(4 \beta \cos u+\gamma(3+\cos 2 u)) \csc ^{2} u \sinh v H,
$$

satisfying

$$
\begin{aligned}
\left\{J_{1}, J_{2}\right\}= & 16 T_{1} T_{3} T_{4}-4(4 \beta \cos u+\gamma(3+\cos 2 u)) \cosh v \csc ^{2} u T_{1} H \\
& -4 \operatorname{sech} v \tanh v \sin u T_{4} p_{w}^{2} .
\end{aligned}
$$

\subsubsection{Reduction using the isometry $K_{3}=f_{3}$}

With $K_{3}=f_{3}$, it is again possible to choose coordinates such that $K_{3}=P_{3}$, and to set the coefficient of $P_{1} P_{2}$ to be zero:

$$
Q_{1}=\frac{q_{2}}{q_{1}}, \quad Q_{2}=\frac{q_{1}^{2}+q_{2}^{2}+q_{3}^{2}}{\sqrt{q_{1}^{2}+q_{2}^{2}}}, \quad Q_{3}=\frac{q_{3}}{2\left(q_{1}^{2}+q_{2}^{2}+q_{3}^{2}\right)},
$$


giving

$$
H=\frac{Q_{1}^{2}}{\beta\left(1+Q_{1}^{2}\right)+\gamma \sqrt{1+Q_{1}^{2}}}\left(\left(1+Q_{1}^{2}\right)^{2} P_{1}^{2}+Q_{2}^{2} P_{2}^{2}+\frac{P_{3}^{2}}{4 Q_{2}^{2}}\right) .
$$

A further change of coordinates

$$
Q_{1}=\tan u, \quad Q_{2}=\mathrm{e}^{v}, \quad Q_{3}=w,
$$

gives

$$
H=\frac{\sin ^{2} u}{\beta+\gamma \cos u}\left(p_{u}^{2}+p_{v}^{2}+\frac{1}{4} \mathrm{e}^{-2 v} p_{w}^{2}\right)
$$

which has the $D_{4}$ kinetic energy, with potential (equivalent to the " $a_{1}$ " part of Case A in [9]).

\section{The conformal algebra and quadratic integrals}

We can see that $K_{3}=f_{3}$ commutes with the 6 functions

$$
2 e_{3} f_{3}+h_{1}^{2}, 4 e_{1} f_{1}-h_{3}^{2}, 8 e_{2} f_{2}-h_{4}^{2}, h_{2}, f_{1} \text {, and } f_{2},
$$

which correspond to the 6-dimensional conformal algebra of our $2 \mathrm{D}$ kinetic energy $H_{0}$. In the present coordinates, these take the form

$$
\begin{aligned}
& T_{1}=p_{v}, \quad T_{2}=\mathrm{e}^{v}\left(p_{u} \sin u+p_{v} \cos u\right), \quad T_{3}=\mathrm{e}^{v}\left(p_{u} \cos u-p_{v} \sin u\right), \\
& T_{4}=p_{u}, \quad T_{5}=\mathrm{e}^{-v}\left(p_{u} \sin u-p_{v} \cos u\right), \quad T_{6}=\mathrm{e}^{-v}\left(p_{u} \cos u+p_{v} \sin u\right) .
\end{aligned}
$$

The element $T_{1}$ is an invariant of the kinetic energy, but the full expression for $2 K_{2} K_{3}-K_{1}^{2}=$ $2 e_{3} f_{3}+h_{1}^{2}$ (the Casimir of the isometry algebra) is a quadratic integral for $H$ :

$$
J_{1}=\frac{1}{4}\left(2 K_{2} K_{3}-K_{1}^{2}\right)=T_{1}^{2}+\frac{1}{4} \mathrm{e}^{-2 v} p_{w}^{2} .
$$

We also have that $\left\{F_{2}, K_{3}\right\}=\left\{F_{3}, K_{3}\right\}=0$ (see Table 8), but $F_{3}=4\left(J_{1}-\beta H\right)$, so is not independent. However $F_{2}$ is independent and takes the form

$$
J_{2}=F_{2}=-4 T_{3} T_{4}-(4 \beta \cos u+\gamma(3+\cos 2 u)) \csc ^{2} u \mathrm{e}^{v} H .
$$

\subsubsection{The relationship between these reductions}

If we consider the $K_{1}$ reduction to have coordinates $(\bar{u}, \bar{v}, \bar{w})$, then combining the transformations, we obtain

$$
u=\bar{u}, \quad v=-2 \bar{w}+\log (\cosh \bar{v}), \quad w=\frac{1}{2} \mathrm{e}^{2 \bar{w}} \tanh \bar{v}
$$

and the Hamiltonian (9.2) is transformed onto that of (9.1) (with bars). This transformation preserves the form of the 2-dimensional kinetic energy, but changes the potential, thus relating two of the potentials obtained in [9]. 


\subsection{The case when $\beta=0$}

We now consider the Hamiltonian

$$
H=\frac{q_{2}^{2} q_{3}^{2} \sqrt{q_{1}^{2}+q_{2}^{2}}}{\alpha q_{2}^{2} \sqrt{q_{1}^{2}+q_{2}^{2}}+\gamma q_{1} q_{3}^{2}}\left(p_{1}^{2}+p_{2}^{2}+p_{3}^{2}\right),
$$

which has the single isometry $K_{1}=h_{1}$. We therefore reduce with respect to this by making an appropriate choice of coordinates $Q_{i}$, which would generally give "vector potential" terms, which again turn out to be gauge equivalent to zero. We can again incorporate this gauge transformation into the definition of $Q_{3}$, to obtain

$$
Q_{1}=\frac{q_{2}}{q_{1}}, \quad Q_{2}=\frac{q_{3}}{\sqrt{q_{1}^{2}+q_{2}^{2}}}, \quad Q_{3}=-\frac{1}{4} \log \left(q_{1}^{2}+q_{2}^{2}+q_{3}^{2}\right),
$$

giving

$$
H=\frac{Q_{1}^{2} Q_{2}^{2}}{\alpha Q_{1}^{2}+\gamma Q_{2}^{2} \sqrt{Q_{1}^{2}+1}}\left(\left(Q_{1}^{2}+1\right)^{2} P_{1}^{2}+\left(Q_{2}^{2}+1\right) P_{2}^{2}+\frac{P_{3}^{2}}{4\left(Q_{2}^{2}+1\right)}\right) .
$$

The final transformation puts the kinetic energy into the form $H_{0}=\tilde{\varphi}(u, v)\left(p_{u}^{2}+p_{v}^{2}\right)$ :

$$
Q_{1}=\tan u, \quad Q_{2}=\sinh v, \quad Q_{3}=w,
$$

giving

$$
H=\frac{\sin ^{2} u \sinh ^{2} v}{\alpha \sin ^{2} u+\gamma \cos u \sinh ^{2} v}\left(p_{u}^{2}+p_{v}^{2}+\frac{1}{4} \operatorname{sech}^{2} v p_{w}^{2}\right) .
$$

The corresponding metric has no Killing vectors, so we cannot reduce further. However, we can construct the 6-dimensional conformal algebra from the linear and quadratic invariants of $h_{1}$ :

$$
h_{2}, h_{3}, h_{4}, e_{1} f_{1}, e_{2} f_{2}, e_{3} f_{3},
$$

giving

$$
\begin{aligned}
& T_{1}=p_{u}, \quad T_{2}=\sin u \sinh v p_{u}+\cos u \cosh v p_{v}, \quad T_{3}=\cos u \sinh v p_{u}-\sin u \cosh v p_{v}, \\
& T_{4}=\sin u \cosh v p_{u}+\cos u \sinh v p_{v}, \quad T_{5}=\cos u \cosh v p_{u}-\sin u \sinh v p_{v}, \quad T_{6}=p_{v} .
\end{aligned}
$$

We see from (5.3) that $h_{1}$ commutes with $F_{3}$ and $F_{1} F_{2}$, which therefore give us a quadratic and a quartic integral for $H$, which can be written in terms of the conformal algebra:

$$
\begin{aligned}
& J_{1}=T_{1}^{2}-\gamma \cot u \operatorname{cosec} u H, \\
& J_{2}=\left(2 T_{1} T_{5}-\gamma \cosh v \operatorname{cosec}^{2} u\left(1+\cos ^{2} u\right) H\right)^{2}-T_{1}^{2} p_{w}^{2} \operatorname{sech}^{2} v \sin ^{2} u .
\end{aligned}
$$

We thus find that the Hamiltonian (9.4) is maximally super-integrable, but that it falls out of the Darboux-Koenigs classification, since it has no first order integral, together with one quadratic and one quartic integral.

\section{Reductions of the system of Section 6.1}

We now consider the Hamiltonian

$$
H=\frac{1}{\alpha\left(q_{1}^{2}+q_{2}^{2}+q_{3}^{2}\right)+\beta q_{1}+\gamma}\left(p_{1}^{2}+p_{2}^{2}+p_{3}^{2}\right)
$$

with symmetry algebra $\left\langle K_{1}, K_{2}, K_{3}\right\rangle$, defined by (6.3a).

We can therefore reduce with respect to either $K_{1}$ or $K_{2}$. Reduction with respect to $K_{3}$ would be equivalent to that with respect to $K_{2}$ through the action of the involution $\iota_{23}$. 


\subsection{Reduction using the isometry $K_{1}=h_{4}$}

To transform $K_{1}=h_{4}$ to $P_{3}$ and to eliminate the $P_{1} P_{2}$ term, we choose coordinates

$$
Q_{1}=q_{1}, \quad Q_{2}=\sqrt{q_{2}^{2}+q_{3}^{2}}, \quad Q_{3}=\frac{1}{4} \arctan \left(\frac{q_{2}}{q_{3}}\right),
$$

giving

$$
H=\frac{1}{\alpha\left(Q_{1}^{2}+Q_{2}^{2}\right)+\beta Q_{1}+\gamma}\left(P_{1}^{2}+P_{2}^{2}+\frac{P_{3}^{2}}{16 Q_{2}^{2}}\right) .
$$

Since $\left\{K_{2}^{2}+K_{3}^{2}, K_{1}\right\}=0$, we use this to build an invariant of (10.1)

$$
K_{2}^{2}+K_{3}^{2}=4\left(T_{1}^{2}+\frac{\left(2 \alpha Q_{1}+\beta\right)^{2}}{16 Q_{2}^{2}} P_{3}^{2}\right), \quad T_{1}=2 \alpha Q_{2} P_{1}-\left(2 \alpha Q_{1}+\beta\right) P_{2},
$$

is a first order integral of the kinetic energy.

The change of coordinates

$$
\bar{u}=\alpha\left(Q_{1}^{2}+Q_{2}^{2}\right)+\beta Q_{1}, \quad \bar{v}=\frac{1}{2 \alpha} \arctan \frac{2 \alpha Q_{1}+\beta}{2 \alpha Q_{2}}, \quad \bar{w}=Q_{3},
$$

gives

$$
T_{1}=p_{\bar{v}}, \quad H=\frac{1}{\bar{u}+\gamma}\left(\left(4 \alpha \bar{u}+\beta^{2}\right) p_{\bar{u}}^{2}+\frac{1}{4 \alpha \bar{u}+\beta^{2}} p_{\bar{v}}^{2}+\frac{\alpha^{2} p_{\bar{w}}^{2}}{4\left(4 \alpha \bar{u}+\beta^{2}\right) \cos ^{2}(2 \alpha \bar{v})}\right) .
$$

With the final step in the transformation,

$$
u=\ln \left(\bar{u}+\frac{\beta^{2}}{4 \alpha}\right), \quad v=4 \alpha \bar{v}, \quad w=\bar{w},
$$

we obtain

$$
T_{1}=4 \alpha p_{v}, \quad H=\frac{16 \alpha^{2} \mathrm{e}^{-u}}{4 \alpha \mathrm{e}^{u}+4 \alpha \gamma-\beta^{2}}\left(p_{u}^{2}+p_{v}^{2}+\frac{p_{w}^{2}}{64 \cos ^{2} \frac{v}{2}}\right),
$$

which has the $D_{3}$ kinetic energy, with potential (equivalent to the " $b_{1}$ " part of Case B in [9]).

\subsubsection{The conformal algebra and quadratic integrals}

We can see that $K_{1}=h_{4}$ commutes with the 6 functions

$$
K_{2}^{2}+K_{3}^{2}, e_{2}^{2}+e_{3}^{2}, f_{2}^{2}+f_{3}^{2}, e_{1}, h_{1} \text {, and } f_{1},
$$

which correspond to the 6 -dimensional conformal algebra of our $2 \mathrm{D}$ kinetic energy $H_{0}$. In the present coordinates, these take the form

$$
\begin{aligned}
& T_{1}=p_{v}, \quad T_{2}=\mathrm{e}^{-\frac{1}{2} u}\left(p_{u} \sin \frac{v}{2}+p_{v} \cos \frac{v}{2}\right), \quad T_{3}=\mathrm{e}^{-\frac{1}{2} u}\left(p_{u} \cos \frac{v}{2}-p_{v} \sin \frac{v}{2}\right), \\
& T_{4}=p_{u}, \quad T_{5}=\mathrm{e}^{\frac{1}{2} u}\left(p_{u} \sin \frac{v}{2}-p_{v} \cos \frac{v}{2}\right), \quad T_{6}=\mathrm{e}^{\frac{1}{2} u}\left(p_{u} \cos \frac{v}{2}+p_{v} \sin \frac{v}{2}\right) .
\end{aligned}
$$

The element $T_{1}$ is an invariant of the kinetic energy, but the full expression for $K_{2}^{2}+K_{3}^{2}$ gives a quadratic integral for $H$ :

$$
J_{1}=T_{1}^{2}+\frac{1}{64} \operatorname{sech}^{2} \frac{v}{2} p_{w}^{2} .
$$

We also have that $\left\{F_{1}, K_{1}\right\}=0$ (see Table 10), giving us

$$
J_{2}=T_{2}^{2}+\left(\beta^{2}-4 \alpha \mathrm{e}^{u} \sin ^{2} \frac{v}{2}\right) H .
$$




\subsection{Reduction using the isometry $K_{2}=\alpha h_{2}+\beta e_{2}$}

To transform $K_{2}=\alpha h_{2}+\beta e_{2}$ to $P_{3}$ and to eliminate the $P_{1} P_{2}$ term, we choose coordinates

$$
Q_{1}=q_{3}, \quad Q_{2}=\sqrt{\alpha q_{1}^{2}+\alpha q_{2}^{2}+\beta q_{1}}, \quad Q_{3}=-\frac{1}{2 \alpha} \arctan \left(\frac{2 \alpha q_{1}+\beta}{2 \alpha q_{2}}\right),
$$

giving

$$
H=\frac{1}{\alpha Q_{1}^{2}+Q_{2}^{2}+\gamma}\left(P_{1}^{2}+\frac{4 \alpha Q_{2}^{2}+\beta^{2}}{4 Q_{2}^{2}} P_{2}^{2}+\frac{P_{3}^{2}}{4 \alpha Q_{2}^{2}+\beta^{2}}\right) .
$$

Since $\left\{K_{2}^{2}+K_{3}^{2}+\frac{\alpha^{2}}{4} K_{1}^{2}, K_{2}\right\}=0$, we use this to build an invariant of (10.2)

$$
K_{2}^{2}+K_{3}^{2}+\frac{\alpha^{2}}{4} K_{1}^{2}=4\left(T_{1}^{2}+\frac{\left(4 \alpha^{2} Q_{1}^{2}+4 \alpha Q_{2}^{2}+\beta^{2}\right)}{4 \alpha Q_{2}^{2}+\beta^{2}} P_{3}^{2}\right),
$$

where $T_{1}=\frac{\sqrt{4 \alpha Q_{2}^{2}+\beta^{2}}}{Q_{2}}\left(Q_{2} P_{1}-\alpha Q_{1} P_{2}\right)$ is a first order integral of the kinetic energy.

The change of coordinates,

$$
\bar{u}=\alpha Q_{1}^{2}+Q_{2}^{2}, \quad \bar{v}=\frac{1}{2 \alpha} \arctan \frac{2 \alpha Q_{1}}{\sqrt{4 \alpha Q_{2}^{2}+\beta^{2}}}, \quad \bar{w}=Q_{3},
$$

gives

$$
T_{1}=p_{\bar{v}}, \quad H=\frac{1}{\bar{u}+\gamma}\left(\left(4 \alpha \bar{u}+\beta^{2}\right) p_{\bar{u}}^{2}+\frac{1}{4 \alpha \bar{u}+\beta^{2}} p_{\bar{v}}^{2}+\frac{p_{\bar{w}}^{2}}{\left(4 \alpha \bar{u}+\beta^{2}\right) \cos ^{2}(2 \alpha \bar{v})}\right) .
$$

With the final step in the transformation,

$$
u=\ln \left(\bar{u}+\frac{\beta^{2}}{4 \alpha}\right), \quad v=4 \alpha \bar{v}, \quad w=\bar{w},
$$

we obtain

$$
T_{1}=4 \alpha p_{v}, \quad H=\frac{16 \alpha^{2} \mathrm{e}^{-u}}{4 \alpha \mathrm{e}^{u}+4 \alpha \gamma-\beta^{2}}\left(p_{u}^{2}+p_{v}^{2}+\frac{p_{w}^{2}}{16 \alpha^{2} \cos ^{2} \frac{v}{2}}\right),
$$

which has the $D_{3}$ kinetic energy, with the same potential (equivalent to the " $b_{1}$ " part of Case B in $[9])$.

\section{Conclusions}

The purpose of this paper was to extend the method developed in [6] from 2 to 3 degrees of freedom. The original motivation for [6] was to understand Darboux-Koenigs super-integrable systems, which are characterised as having exactly one first order integral and 2 second order integrals. These are therefore associated with non-constant curvature metrics, which, in 2 dimensions, are necessarily conformally flat. The method of [6] exploited the conformal flatness by building first integrals from conformal symmetries.

In the current paper we considered 3-dimensional conformally flat metrics and built integrals from the corresponding conformal algebra (2.2). We initially assumed one first order integral $K$ and three quadratic ones $F_{i}$, giving us five integrals in all. A further requirement was involutivity of three of these and independence of them all, to give us a super-integrable system. These 
requirements are the obvious generalisation of the Darboux-Koenigs systems to 3 degrees of freedom.

The classification of such systems is a difficult task. As can be seen in the examples, we could start with a different collection of quadratic integrals to arrive at the same Hamiltonian (in particular, for the cases with larger symmetry algebras). However, the structure of the conformal algebra (2.2), with its involutive automorphisms, enabled us to limit the starting choice of Killing vector to either $e_{1}, h_{1}$ or $h_{4}$, thus reducing the overall number of choices. We did not tackle the classification problem, but presented a number of interesting, multi-parameter examples, which, upon restriction, led to systems with a higher degree of symmetry, which were then able to be reduced to 2 degrees of freedom. Almost all our examples, upon reduction, had one linear and two quadratic first integrals, so were inevitably in the Darboux-Koenigs classification. However, the third dimension is "remembered" through the addition of a potential function, all of which were just special cases of those classified in $[9,10]$. Furthermore, when there existed two inequivalent symmetries (with respect to involutions of the system under study) we were able to reduce in two different ways, leading to the introduction of two different potentials, and the two potentials could be related through a transformation in the 3 -dimensional space. This means that the potentials in the classification of $[9,10]$ are not fully independent, if we allow transformations which take us out of the 2-dimensional space.

We see from the results of Section 7, that different parameter restrictions of the 3-parameter Hamiltonian with conformal factor (4.3) can reduce to different Darboux-Koenigs cases. This means that the Hamiltonian (4.1), with $\varphi$ given by (4.3), simultaneously contains both DarbouxKoenigs cases $D_{2}$ and $D_{4}$. Furthermore, since there are only 4 metrics in the Darboux-Koenigs classification, we inevitably arrive at the same metric, from two or more different 3 -dimensional metrics (see the examples of Sections 7.2 and 9.1).

Whilst most of our examples (before reduction) possessed a 3-dimensional isometry algebra, the Hamiltonian (9.3) had only one first order integral, so the reduced metric had no Killing vectors at all. Nevertheless, we were able to show that the reduced Hamiltonian was maximally super-integrable, possessing quadratic and quartic integrals, thus falling out of the DarbouxKoenigs class of metric.

This paper represents an important first step in our investigations into the use of the conformal algebra to build higher order first integrals. Clearly we need a better understanding of the underlying structure in order to tackle the important open problem of the classification of such higher-dimensional superintegrable systems and their reductions.

Reducing from 3 to 2 dimensions, we acquired 1 parameter potentials. It is likely that if we start from a 4-dimensional system, with a large enough isometry algebra, we could produce a 2-parameter restriction of the potentials given in $[9,10]$. Perhaps the full potential could be recovered by going to high enough dimensions.

\section{Acknowledgements}

This work was supported by NSFC (Grant No. 11871396) and NSF of Shaanxi Province of China (Grant No. 2018JM1005). APF thanks Boris Kruglikov for useful discussions on the "gap problem". We thank the referees and an editor for their useful remarks.

\section{References}

[1] Ballesteros A., Enciso A., Herranz F.J., Ragnisco O., Superintegrability on $N$-dimensional curved spaces: central potentials, centrifugal terms and monopoles, Ann. Physics 324 (2009), 1219-1233, arXiv:0812.1882.

[2] Ballesteros A., Enciso A., Herranz F.J., Ragnisco O., Riglioni D., Quantum mechanics on spaces of nonconstant curvature: the oscillator problem and superintegrability, Ann. Physics 326 (2011), 2053-2073, arXiv:1102.5494. 
[3] Dubrovin B.A., Fomenko A.T., Novikov S.P., Modern geometry - methods and applications, Vols. 1-3, Springer-Verlag, New York, 1984.

[4] Escobar-Ruiz M.A., Miller Jr. W., Toward a classification of semidegenerate 3D superintegrable systems, J. Phys. A: Math. Theor. 50 (2017), 095203, 22 pages, arXiv:1611.02977.

[5] Fordy A.P., A Kaluza-Klein reduction of super-integrable systems, J. Geom. Phys. 131 (2018), 210-219, arXiv:1801.02981.

[6] Fordy A.P., First integrals from conformal symmetries: Darboux-Koenigs metrics and beyond, arXiv:1804.06904.

[7] Fordy A.P., Huang Q., Poisson algebras and 3D superintegrable Hamiltonian systems, SIGMA 14 (2018), 022, 37 pages, arXiv:1708.07024.

[8] Gilmore R., Lie groups, Lie algebras, and some of their applications, Wiley, New York, 1974.

[9] Kalnins E.G., Kress J.M., Miller Jr. W., Winternitz P., Superintegrable systems in Darboux spaces, J. Math. Phys. 44 (2003), 5811-5848, arXiv:math-ph/0307039.

[10] Kalnins E.G., Kress J.M., Winternitz P., Superintegrability in a two-dimensional space of nonconstant curvature, J. Math. Phys. 43 (2002), 970-983, arXiv:math-ph/0108015.

[11] Koenigs G.X.P., Sur les géodésiques a integrales quadratiques, in Le cons sur la théorie générale des surfaces, Vol. 4, Editor J.G. Darboux, Chelsea Publishing, New York, 1972, 368-404.

[12] Kruglikov B., The D., The gap phenomenon in parabolic geometries, J. Reine Angew. Math. 723 (2017), 153-215, arXiv:1303.1307.

[13] Matveev V.S., Shevchishin V.V., Two-dimensional superintegrable metrics with one linear and one cubic integral, J. Geom. Phys. 61 (2011), 1353-1377, arXiv:1010.4699.

[14] Miller Jr. W., Post S., Winternitz P., Classical and quantum superintegrability with applications, J. Phys. A: Math. Theor. 46 (2013), 423001, 97 pages, arXiv:1309.2694.

[15] Valent G., Superintegrable models on Riemannian surfaces of revolution with integrals of any integer degree (I), Regul. Chaotic Dyn. 22 (2017), 319-352, arXiv:1703.10870. 